\title{
PERSPECTIVAS ARQUEOLÓGICAS PARA PATAGONIA SEPTENTRIONAL: SITIO CUEVA HUENUL 1 (PROVINCIA DEL NEUQUÉN, ARGENTINA)
}

RAMIRO BARBERENA", KAREN BORRAZZO"*, AGUSTINA A. RUGHINI"**, GUADALUPE ROMERO ${ }^{\alpha}$, M. PAZ POMPEI ${ }^{\beta}$, CARINA LLANO", M. EUGENIA DE PORRAS ${ }^{\pi}$, VÍCTOR DURÁN", CHARLES R. STERN ${ }^{2}$, ANAHÍ RE ${ }^{\alpha}$, DIEGO ESTRELLA ${ }^{*}$, ANALÍA FORASIEPI ${ }^{\varepsilon}$, FERNANDO J. FERNÁNDEZ ${ }^{r}$, MANUEL CHIDIAK", LUIS ACUÑA*, ALEJANDRA GASCO* \& MARÍA NELLA QUIROGA*

\section{RESUMEN}

El norte de la provincia de Neuquén (Depto. Pehuenches, Argentina) es muy poco conocido a nivel arqueológico, a pesar de estar ubicado en una posición central en relación con distintos temas clave del poblamiento humano de Sudamérica, incluyendo la extinción de la megafauna y sus causas, el poblamiento humano inicial y la existencia de discontinuidades arqueológicas en el Holoceno medio. En este trabajo se presenta el primer cuerpo de resultados paleoecológicos y arqueológicos para el sitio Cueva Huenul 1, recientemente excavado, que ofrece una secuencia sedimentaria que se extiende durante los últimos 16.000 años calendáricos. Estas evidencias incluyen el desarrollo de un marco crono-estratigráfico para el sitio, que aporta novedosa información tefro-cronológica. A partir de este análisis, se definen cuatro componentes temporales, sobre los cuales se asienta el estudio de los materiales recuperados: evidencias faunísticas (paleontológicas y arqueológicas), arqueobotánicas, líticas, cerámicas y de arte rupestre. Estos resultados en escala de sitio proveen una primera instancia de evaluación de procesos en escala macro-regional, así como las bases para la continuación de este proyecto.

PALABRAS CLAVE: Neuquén, fauna extinta, poblamiento humano temprano, cronología, tendencias temporales.

\section{ARCHAEOLOGICAL PERSPECTIVES FOR NORTHERN PATAGONIA: CUEVA HUENUL 1 SITE (NEUQUEN PROVINCE, ARGENTINA)}

\author{
ABSTRACT \\ Northern Neuquén Province (Pehuenches Dept., Argentina) is barely known from an archaeological \\ CONICET, Laboratorio de Paleoecología Humana, Facultad de Ciencias Exactas y Naturales, Universidad Nacional de Cuyo, \\ Mendoza, Argentina. ramidus28@gmail.com \\ * CONICET - IMHICIHU, FFyL - Universidad de Buenos Aires. Buenos Aires, Argentina. \\ Universidad de Buenos Aires - FFyL. Buenos Aires, Argentina. \\ a CONICET, UBA, INAPL. Buenos Aires, Argentina. \\ в Museo de Historia Natural de San Rafael, San Rafael, Argentina. \\ $\pi \quad$ CEAZA. La Serena, Chile. \\ $\Omega \quad$ University of Colorado, Department of Geological Sciences. Boulder, EE.UU. \\ $\varepsilon \quad$ CONICET - CRICYT. Mendoza, Argentina. \\ r CONICET, Universidad Nacional de La Plata. La Plata, Argentina.
}


perspective, though it is centrally placed in terms of several large-scale key issues in the peopling of South America: the extinction of the megafauna and its causes, early human presence, and the existence of archaeological discontinuities during the Mid-Holocene. In this paper we present the first body of paleoecological and archaeological data for Cueva Huenul 1 site, recently excavated, which offers a sedimentary sequence extending during the last of 16.000 calendar years. Initially, we present a chronostratigraphic frame for the site, including new tephro-chronological information. On this basis, four temporal components are defined, providing the historical scheme for the analysis of the recovered evidences that include: archaeofaunas (paleontological and archaeological), archaeobotany, lithic and ceramic technology, and rockart. These results at a site scale provide a first approach to a discussion of macro-regional processes, as well as the basis for the continuation of our research.

KEY WORDS: Neuquén, extinct fauna, early human peopling, chronology, temporal tendencies.

\section{INTRODUCCIÓN Y ANTECEDENTES}

Este trabajo se enmarca en un proyecto arqueológico recientemente iniciado en el extremo noroeste de Patagonia (Departamento Pehuenches, provincia del Neuquén, Argentina; Fig. 1; Barberena, 2013). Básicamente la región carece de información arqueológica, lo que ofrece tanto una limitación como un desafío para el desarrollo de este proyecto. En esta instancia inicial de acercamiento a la arqueología regional, nos interesa evaluar en escala microregional el lugar que ocupó la región en el marco de la organización geográfica y la demografía humana en el extremo norte de Patagonia. A nivel temporal el acercamiento abarca todo el lapso del poblamiento humano, e incluye preguntas de amplia discusión reciente: ¿Existió algún tipo de interacción entre las sociedades humanas tempranas y la megafauna pleistocénica? ¿Se expresa a nivel local la discontinuidad demográfica registrada durante el Holoceno medio en diversas regiones de Sudamérica? ¿Fue la región ocupada en forma marginal durante el

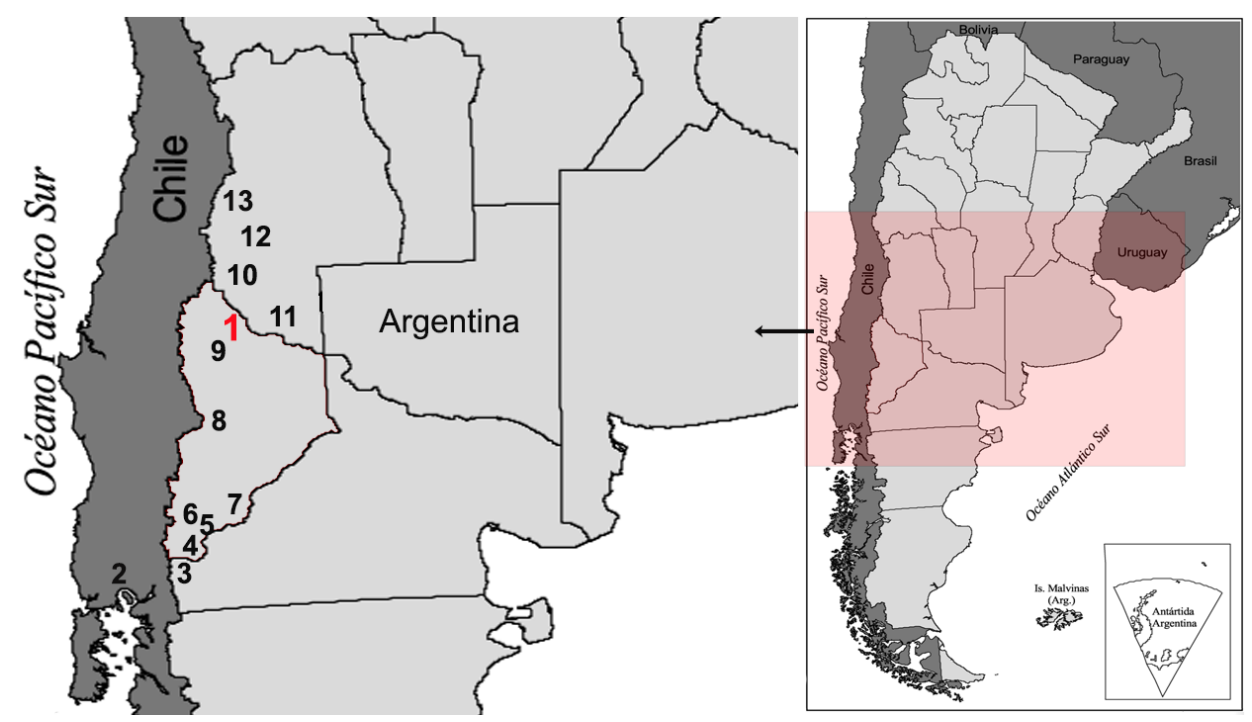

Fig. 1. Ubicación del sitio $\mathrm{CH} 1$ y otros sitios mencionados en el texto.
Referencias: 1) $\mathrm{CH} 1$, 2) Monte Verde, 3) El Trébol, 4) Arroyo Corral II, 5) Cuyín Manzano, 6) Cueva Traful I, 7) Cueva Epullán Grande, 8) Cueva Haichol, 9) Aquihuecó y Hermanos Lazcano, 10) Gruta El Manzano, 11) Campo volcánico La Payunia, 12) El Chacay, 13) Arroyo Malo 3.
1 En las localidades de Barrancas y Buta Ranquil este sitio es conocido bajo los nombres de Alero Tracaicó y Cueva Huemul (sic) respectivamente, aunque en la bibliografía arqueológica es tradicionalmente conocido como Cueva Huenul, término tomado de la toponimia regional en lengua Mapudungun: [Huenul] Denominación muy frecuente de cerros de altura relativa mediana y de forma redondeada de flancos lisos, compuestos de material blando. Se aplica especialmente a pequeños volcanes basálticos del sud de Mendoza y del norte de Neuquén, pero no faltan otros de composición distinta (Groeber 1926:58). 


\section{Holoceno tardío?}

Se presenta una síntesis del cuerpo de resultados producto de la excavación y análisis de los materiales del sitio Cueva Huenul 1 (de aquí en adelante $\mathrm{CH} 1)^{1}$, incluyendo conjuntos líticos, faunísticos, arqueobotánicos y de arte rupestre. Este trabajo expande publicaciones preliminares previas (Barberena et al. 2010; Pompei et al. 2012) y se basa sobre un estudio crono-estratigráfico y formacional del sitio donde se presenta la información estratigráfica detallada (Barberena, 2014). Este sitio constituye el primer bloque de información con contenido cronológico disponible a nivel regional. Esto marca la necesidad de integrar las evidencias de regiones vecinas, las cuales permitirán aproximarnos a la escala espacial de los procesos demográficos del pasado.

$\mathrm{CH} 1$ ocupa un lugar importante en la historia de las investigaciones arqueológicas en el noroeste de Patagonia, aunque se encontraba virtualmente inédito. En el año 1978, Jorge Fernández realizó la primera campaña de excavaciones en el sitio, en conjunto con Juan Schobinger, Rafael Goñi, Marta Ruiz y Carlos Baied. Como producto de estos trabajos pioneros se realizó una fecha radiocarbónica sobre materia orgánica de procedencia actualmente desconocida, la cual arrojó una edad de $11.150 \pm 230$ años ${ }^{14} \mathrm{C}$ AP (Cordero et al. 2002). Aunque se carece de información contextual publicada, esta muestra indicó la presencia de sedimentos estratificados correspondientes al Pleistoceno tardío. Se han dado a conocer brevemente las pinturas rupestres presentes en las paredes internas de la cueva (Fernández 1974-1976; Schobinger 1985). En una escala regional contamos con las investigaciones realizadas por Hajduk, Cúneo, Della Negra y equipo en la cuenca del río Curi Leuvú ubicada al sur de CH1 (Della Negra et al. 2009; Hajduk \& Cúneo, 2009; Hajduk et al. 2011). En escala macroregional se cuenta con valiosos antecedentes para las regiones vecinas: sur de Mendoza, incluyendo la cuenca del río Grande y el campo volcánico La Payunia (Durán, 2000; Gil, 2006; Salgán, 2012), centro-sur de Neuquén (Fernández, 1988-1990; Crivelli Montero et al. 1996; López et al. 2009), La Pampa (Berón, 2007) y centro-sur de Chile, particularmente las regiones de la Araucanía y el Maule (Menghin, 1962; Dillehay, 2010; Massone et al. 2011; Campbell \& Quiroz, 2015).

El sitio se ubica en $36^{\circ} 57^{\prime} \mathrm{S}, 6^{\circ} 49^{\prime} \mathrm{O}$, a $1000 \mathrm{msnm}$, en una posición adyacente al ecotono entre las comunidades fitogeográficas del Monte y Patagonia. La vegetación de la región se encuentra dominada por estepas arbustivas de transición compuestas por Verbenaceae, Asteraceae subf. Asteroideae, Larrea, Prosopis y Prosopidastrum (Movia et al. 1982). En este sector de la Patagonia, comprendido entre los $30^{\circ}-40^{\circ} \mathrm{S}$, ocurre la transición de los sistemas de circulación atmosférica Subtropical y Templado (Páez et al. 2004), lo que se traduce en un clima árido y semiárido. Tomando los patrones climáticos Norte y Sur descriptos por Páez et al. (2004), el sitio se encuentra bajo la influencia del patrón Sur. Este refleja una variabilidad en la estacionalidad de las precipitaciones, cuyas isoyetas están entre 200$300 \mathrm{~mm}$ anuales a una altura de $1000 \mathrm{msnm}$, en tanto que por debajo de esta altura la precipitación media anual es menor a $200 \mathrm{~mm}$.

\section{MATERIALES Y MÉTODOS}

\section{Materiales}

$\mathrm{CH} 1$ es una cueva de grandes dimensiones originada por procesos erosivos, principalmente de carácter hídrico, que actuaron en el punto de contacto entre dos litologías de diferente tenacidad: las ignimbritas correspondientes a la Formación Tilhué en la base, constituyendo las paredes y la roca de base de la cueva, y una colada basáltica que corresponde a la Formación El Puente, que conforma el techo de la misma (Narciso et al. 2004; Fig. 2). La circulación de agua es un proceso que tiene una importancia central en la génesis y evolución del reparo y en la preservación de los depósitos sedimentarios contenidos en su interior. Hemos registrado evidencias de filtración recurrente de agua en una línea que atraviesa la cueva en su sector central (Fig. 2). De este modo, los depósitos se encuentran expuestos a la acción de dos líneas de goteo paralelas: una línea de goteo 'interna', en el centro del reparo, y una línea de goteo 'externa', en la boca del mismo (la línea de goteo en sentido estricto). Esto condiciona la preservación diferencial de los depósitos estratificados: el sector ubicado por detrás de la línea de goteo interna 

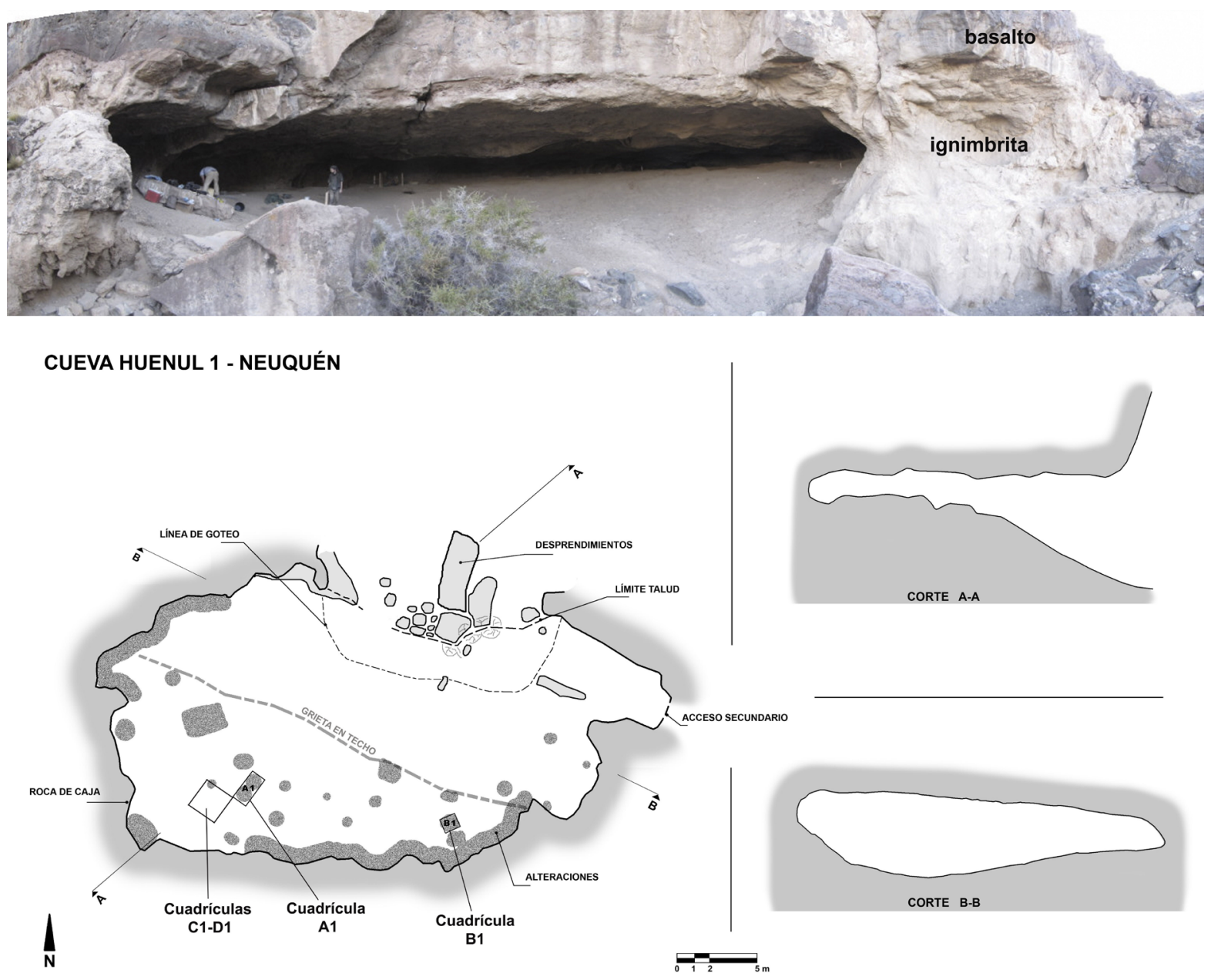

Fig. 2. Imagen y planta del sitio $\mathrm{CH} 1$.

tiene un depósito sedimentario potente y baja erosión hídrica, mientras que el amplio sector ubicado entre las líneas de goteo interna y externa se encuentra expuesto a erosión hídrica intensa, con una consecuente redepositación y pérdida de los sedimentos hacia el exterior del reparo (perfil en Fig. 2).

Se realizaron excavaciones en dos sectores diferentes de la cueva a fin de evaluar la variación estratigráfica (Fig. 2). En el sector más profundo de la cueva se emplazaron tres cuadrículas de 2 x 1 m: A1 fue excavada en 2009 y presentó una profundidad máxima de $1.4 \mathrm{~m}\left(2.8 \mathrm{~m}^{3}\right.$ de volumen excavado), C1 y D1 fueron excavadas en 2012 y se alcanzó una profundidad máxima de 1 $\mathrm{m}$ (4 $\mathrm{m}^{3}$ de volumen excavado). En una posición más cercana a la boca de la cueva se excavó en 2009 la cuadrícula B1 con una dimensión de 1 x $1 \mathrm{~m}$ y una profundidad de $0.95 \mathrm{~m}(0.95$ $\mathrm{m}^{3}$ ). La cuadrícula A1 proveyó la secuencia más extensa y menos impactada por procesos postdepositacionales, por lo que fue seleccionada para construir una secuencia crono-estratigráfica inicial y realizar análisis granulométricos, geoquímicos, polínicos y arqueobotánicos. Aquí se presenta el análisis de los materiales procedentes de esa cuadrícula.

\section{Métodos}

La excavación se realizó empleando niveles artificiales de extracción de $10 \mathrm{~cm}$ de potencia, los cuales se numeraron a partir de 1 desde la superficie hacia la base de la excavación. Se 
diferenciaron unidades estratigráficas naturales y rasgos sedimentarios -antrópicos o naturales- en función de criterios litológicos. Sobre la base de criterios estratigráficos y cronológicos se definen componentes estratigráficos, que constituyen los lapsos principales de formación del depósito sedimentario. Para la comparación de frecuencias de materiales se emplean tanto los niveles artificiales de extracción como los componentes temporales (Grayson, 1984; Stein, 1990).

Las fechas radiocarbónicas presentadas son de tipo AMS y fueron obtenidas en el laboratorio National Science Foundation AMS Laboratory, University of Arizona (EEUU). Las mismas fueron calibradas en el programa OxCal 4.2 utilizando la curva IntCal 13 (Reimer et al. 2013) y se grafican mediante OxCal 4.2 (Bronk Ramsey, 2009, 2013). Se emplean dos desvíos estándar, con la excepción de aquellos casos en que, debido a la morfología de la curva de calibración, un solo desvío estándar otorga una probabilidad que es $\geq 0.95$ de interceptar la edad real del evento datado (Bronk Ramsey, 2009). Todas las edades mencionadas en el texto son calibradas AP.

Las muestras de tefra fueron examinadas con un microscópico petrográfico para determinar el color, la morfología y la composición mineralógica del vidrio volcánico. Luego, estas muestras fueron lavadas en agua y acetona para remover las arcillas, molidas a un polvo fino, disueltas en ácido hidroflórico y analizadas mediante técnicas de ICP-MS para caracterizar la composición a nivel de elementos traza en la Universidad de ColoradoBoulder (EEUU).

Los métodos empleados para la recuperación de restos macro-botánicos fueron la flotación y el cernido en seco del sedimento. El uso de tamices con mallas metálicas de $1 \mathrm{~mm}$ de luz y lupa de bajos aumentos permitió una recuperación exhaustiva de materiales de muy pequeño tamaño. Aquí se utiliza la información proveniente de los restos recuperados por cernido en seco. La identificación de los restos se llevó a cabo por comparación cualitativa y cuantitativa de caracteres macroscópicos de diagnóstico encontrados con los homólogos de las especies de referencia. La descripción de los especímenes se realizó a ojo desnudo y con microscopio estereoscópico (Nikon SMZ800), teniendo en cuenta los caracteres cualitativos y cuantitativos (Llano \& Barberena, 2013).

Los conjuntos óseos de micro-fauna fueron estudiados por Fernando Fernández, Ulyses Pardiñas y Pablo Teta y ya han sido publicados (Fernández et al. 2011, 2012). Estos datos son integrados en la discusión. Los conjuntos de macro-fauna son estudiados desde una perspectiva tafonómica y zooarqueológica, incluyendo las siguientes variables: huellas antrópicas, marcas de carnívoros y roedores, improntas de raíces, meteorización y procesos químicos varios. Para la determinación anatómica se emplearon lineamientos estándar, priorizando la información ofrecida por el NISP (Grayson, 1984; Mengoni Goñalons, 1999). La excelente preservación de los materiales orgánicos permitió recuperar una gran cantidad de coprolitos asignados a diversos taxones, los cuales están siendo estudiados desde una perspectiva paleoparasitológica (Beltrame et al. 2011, 2013). Se presenta una cuantificación por peso de la abundancia de coprolitos de carnívoros pequeños (i.e. zorros).

Los criterios utilizados para el relevamiento de las representaciones se basaron en los propuestos por Gradin (1978) y Aschero (1988) y revisados por $\mathrm{Re}$ (2010). La documentación del arte rupestre se realizó a partir de fotografías digitales y dibujos a mano alzada. Los motivos fueron descriptos en fichas, consignándose allí también los procesos de deterioro naturales y/o antrópicos que los afectan. Una vez en el laboratorio, las imágenes obtenidas fueron procesadas mediante los programas Adobe Photoshop y D-Stretch/Image J, para mejorar la visualización de los motivos.

El análisis tecno-morfológico de los conjuntos artefactuales se realizó siguiendo la propuesta de Aschero (1983) y las modificaciones realizadas por Franco (2002) y Aschero y Hocsman (2004). La identificación de las materias primas líticas fue realizada macroscópicamente por comparación con muestras de mano que fueron descriptas e identificadas petrográficamente (cortes delgados). Para el caso de la obsidiana, materia prima que domina la muestra y se encuentra disponible localmente, se cuenta con información geoquímica de Fluorescencia de Rayos X y Activación Neutrónica obtenida en estudios previos (Barberena et al. 2011). 


\section{RESULTADOS}

Cronología, estratigrafía y procesos de formación del registro

Las cuadrículas A1, C1 y D1 están compuestas por dos conjuntos de unidades litoestratigráficas: desde la roca de base hacia la superficie, las unidades VIII a V tienen elevados porcentajes de materia orgánica y presentan abundantes coprolitos de herbívoros de gran tamaño y materia orgánica vegetal disgregada, que conforma una parte importante de la matriz de estas unidades. Se recuperó un importante número de coprolitos muy bien preservados, cuyo tamaño y morfología sugieren que corresponden a un taxón de la megafauna (Fig. 3). Los coprolitos resultan comparables a los

Tabla 1. Fechados radiocarbónicos del sitio $\mathrm{CH} 1$.

\begin{tabular}{|c|c|c|c|c|c|c|c|}
\hline Muestra & Procedencia & Unidad & Material & Edad ${ }^{14} \mathrm{C}$ & $\begin{array}{c}\text { Edad calibrada } \\
\text { A.P. }\end{array}$ & $\begin{array}{l}\text { Mediana } \\
\text { (cal A.P.) }\end{array}$ & Código \\
\hline \multicolumn{8}{|c|}{ Cuadrículas A1-C1 } \\
\hline CH1.6 & $\begin{array}{l}\text { A1. Nivel } 10 \\
(10-20 \mathrm{~cm})\end{array}$ & I & $\begin{array}{c}\text { Lama guanicoe } \\
\text { (metapodio) }\end{array}$ & $373 \pm 43$ & $506-315$ & 427 & AA 99102 \\
\hline $\mathrm{CH} 1.4$ & $\begin{array}{c}\text { A1. Nivel 2E } \\
(20-30 \mathrm{~cm})\end{array}$ & II & gramíneas (camada) & $1.416 \pm 37$ & $1379-1285$ & 1322 & AA 85721 \\
\hline $\mathrm{CH} 1.7$ & $\begin{array}{l}\text { A1. Nivel 3E } \\
(30-40 \mathrm{~cm})\end{array}$ & III & $\begin{array}{l}\text { Lama guanicoe } \\
\text { (tibia) }\end{array}$ & $1.590 \pm 46$ & $1569-1375$ & 1475 & AA 99103 \\
\hline $\mathrm{CH} 1.16$ & $\mathrm{C} 1$ & $\begin{array}{c}\text { estructura } \\
\text { G }\end{array}$ & $\begin{array}{l}\text { Senna aphylla } \\
\text { (ramas) }\end{array}$ & $4.786 \pm 46$ & $5603-5330$ & 5517 & $\begin{array}{c}\mathrm{AA} \\
102575 \\
\end{array}$ \\
\hline $\mathrm{CH} 1.8$ & $\begin{array}{l}\text { A1. Nivel } 40 \\
(40-50 \mathrm{~cm})\end{array}$ & IV & $\begin{array}{l}\text { Lama guanicoe } \\
\text { (radioulna) }\end{array}$ & $9.375 \pm 91$ & $11068-10277$ & 10601 & AA 99104 \\
\hline $\mathrm{CH} 1.9$ & $\begin{array}{l}\text { A1. Nivel 4E } \\
(40-50 \mathrm{~cm})\end{array}$ & IV & $\begin{array}{l}\text { Lama guanicoe } \\
\text { (húmero) }\end{array}$ & $9.295 \pm 90$ & 10699-10250 & 10483 & AA 99105 \\
\hline $\mathrm{CH} 1.15$ & $\begin{array}{l}\text { A1. Nivel 5E } \\
(50-60 \mathrm{~cm})\end{array}$ & IV & $\begin{array}{l}\text { Prosopis sp. } \\
\text { (semilla) }\end{array}$ & $9.402 \pm 60$ & $11057-10433$ & 10633 & $\begin{array}{c}\text { AA } \\
102574 \\
\end{array}$ \\
\hline $\mathrm{CH} 1.1$ & $\begin{array}{l}\text { A1. Nivel } 50 \\
(55-57 \mathrm{~cm})\end{array}$ & IV & $\begin{array}{c}\text { carbón } \\
\text { (fogón \#2) }\end{array}$ & $9.531 \pm 39$ & 11083-10696 & 10872 & AA 85718 \\
\hline $\mathrm{CH} 1.10$ & $\begin{array}{l}\text { A1. Nivel } 50 \\
(50-60 \mathrm{~cm})\end{array}$ & IV & $\begin{array}{l}\text { Lama guanicoe } \\
\text { (metapodio) }\end{array}$ & $\begin{array}{l}10.155 \\
\pm 98\end{array}$ & 12153-11327 & 11798 & AA 99106 \\
\hline $\mathrm{CH} 1.11$ & $\begin{array}{l}\text { A1. Nivel } 50 \\
(50-60 \mathrm{~cm})\end{array}$ & IV & $\begin{array}{c}\text { Retanilla } \\
\text { patagonica (fruto) }\end{array}$ & $9.261 \pm 66$ & $10588-10250$ & 10436 & AA 99100 \\
\hline CH1.3 & $\begin{array}{l}\text { A1. Nivel } 50 \\
(50-60 \mathrm{~cm})\end{array}$ & V & $\begin{array}{c}\text { coprolito } \\
\text { (megafauna) }\end{array}$ & $\begin{array}{c}11.841 \\
\pm 56\end{array}$ & $13777-13545$ & 13658 & AA 85720 \\
\hline $\mathrm{CH} 1.5$ & $\begin{array}{l}\text { A1. Nivel } 100 \\
(100-110 \mathrm{~cm})\end{array}$ & VII & $\begin{array}{c}\text { coprolito } \\
\text { (megafauna) }\end{array}$ & $\begin{array}{c}13.844 \\
\pm 75 \\
\end{array}$ & $17035-16461$ & 16759 & AA 85722 \\
\hline \multicolumn{8}{|c|}{ Sondeo B1 } \\
\hline $\mathrm{CH} 1.12$ & Nivel 2 & I & $\begin{array}{l}\text { Lama guanicoe } \\
\text { ( húmero ) }\end{array}$ & $1.269 \pm 46$ & $1290-1075$ & 1212 & AA 99109 \\
\hline $\mathrm{CH} 1.14$ & Nivel 5 & III & $\begin{array}{l}\text { Lagenaria sp. } \\
\text { (calabaza) }\end{array}$ & $541 \pm 42$ & $645-508$ & 554 & $\begin{array}{c}\text { AA } \\
102573\end{array}$ \\
\hline $\mathrm{CH} 1.13$ & Nivel 6 & III & $\begin{array}{l}\text { Lama guanicoe } \\
\text { ( radioulna) }\end{array}$ & $1753 \pm 47$ & $1811-1560$ & 1664 & AA 99110 \\
\hline
\end{tabular}


descriptos para Pilosa (Martínez et al. 2010). Se realizaron análisis de $\mathrm{ADN}$ sobre dos muestras de los mismos en McMaster University (Canadá), aunque no se pudo recuperar una señal genética preservada. En la Tabla 1 se presenta el conjunto de edades ${ }^{14} \mathrm{C}$ disponibles para $\mathrm{CH} 1$. Los resultados estratigráficos, granulométricos y geoquímicos han sido presentados en otro trabajo (Barberena, 2014).

Las fechas ${ }^{14} \mathrm{C} \mathrm{CH} 1.3$ y $\mathrm{CH} 1.5$, realizadas sobre coprolitos de megafauna, permiten acotar temporalmente a las unidades VIII-V entre las medianas calibradas de 16.759 y 13.658 años AP. Las unidades basales (VIII-V) están acotadas en su parte superior por una discordancia erosiva que afecta toda la cuadrícula A1 (Fig. 3). Esta zona de contacto presenta evidencias localizadas de redepositación de sedimentos, probablemente por la acción de roedores fosoriales (Ctenomys) o por las ocupaciones humanas posteriores. Estos procesos afectan principalmente a la unidad estratigráfica V. En el perfil Este de la cuadrícula A1 y en las cuadrículas adyacentes C1 y D1 se registró una lámina de unos $2 \mathrm{~cm}$ de espesor conformada por ceniza volcánica apoyando por sobre esta discordancia, denominada Tefra 1 (T1). Su posición permite acotar su edad entre fechas de 13.658 y 11.798 años AP. Se registró una segunda lámina de ceniza volcánica en las cuadrículas C1 y D1, por debajo de la anterior, denominada Tefra 2 (T2).

En contacto directo con la discordancia, se ubica el segundo conjunto de unidades estratigráficas: IV-I. Las mismas se diferencian claramente con respecto al conjunto inferior en términos de color y composición general, ya que no presentan aporte orgánico producto de la disgregación de los coprolitos de megafauna. Seis muestras procedentes de la unidad IV arrojan edades entre 11.798 y 10.436 AP (Tabla 1). Este conjunto establece que la unidad IV (niveles artificiales de extracción 5 y 6), con unos $20 \mathrm{~cm}$ de potencia, es asignable al Holoceno temprano.

Se registran estructuras sedimentarias holocénicas, tales como camadas de gramíneas, un hornillo y dos pozos rellenos con material vegetal, que afectan el sector superior del depósito pleistocénico. Una de las estructuras con material vegetal, denominada $G$ y ubicada en la cuadrícula D1, se encontraba rellena con abundante material vegetal determinado como el taxón de monte Senna aphylla, el cual fue datado en el intervalo 5.600-5.300 AP. Esta estructura constituye la única evidencia correspondiente al Holoceno medio en $\mathrm{CH} 1$. Por último, las unidades III a I corresponden al Holoceno tardío, más específicamente los últimos 1.500 años. En la Fig. 4 se presenta una síntesis de la información geoquímica y granulométrica.

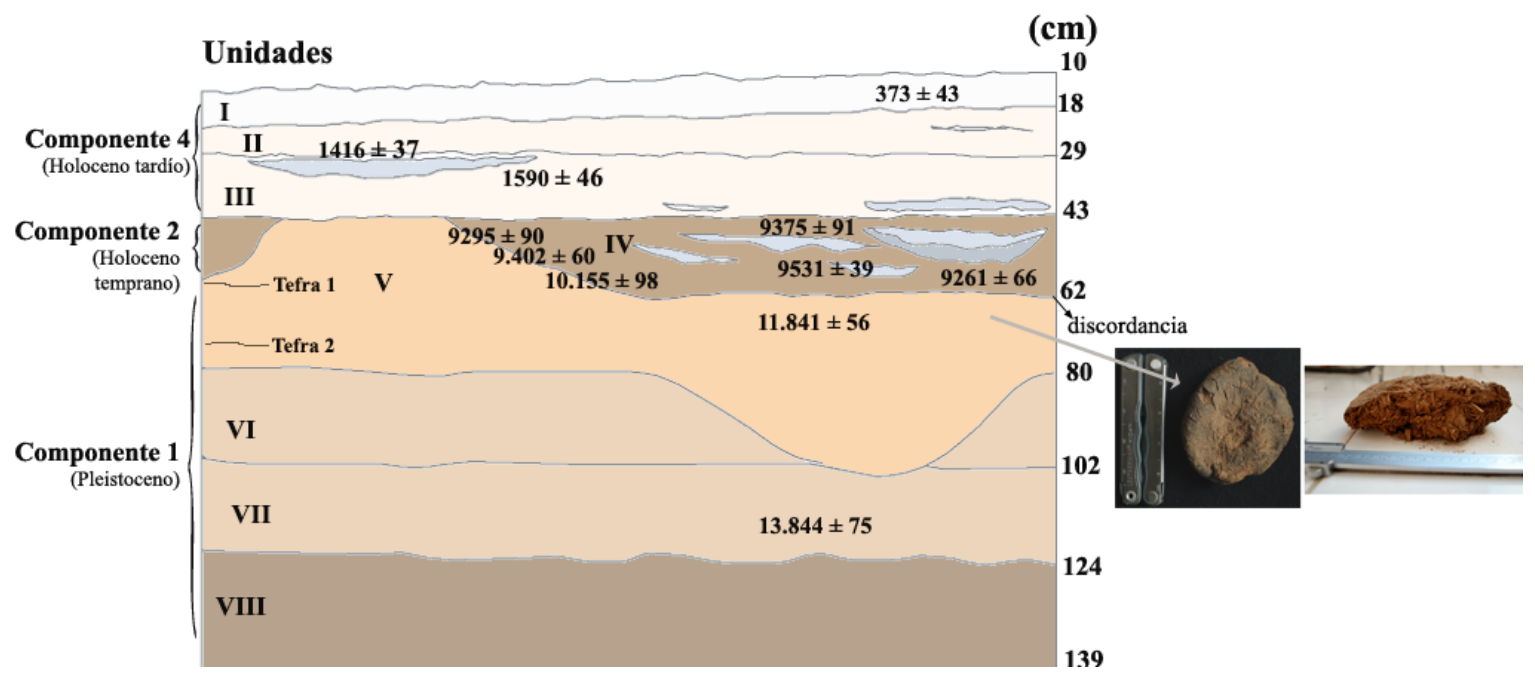

Fig. 3. Perfil Sur de la cuadrícula A1 de $\mathrm{CH} 1$ y coprolitos de megafauna (escalas $=10 \mathrm{~cm}$ ).

Nota: las tefras no fueron registradas en este perfil. Su posición en el esquema es indicativa y se basa en que subyacen a la unidad IV y están contenidas dentro de la unidad V. 


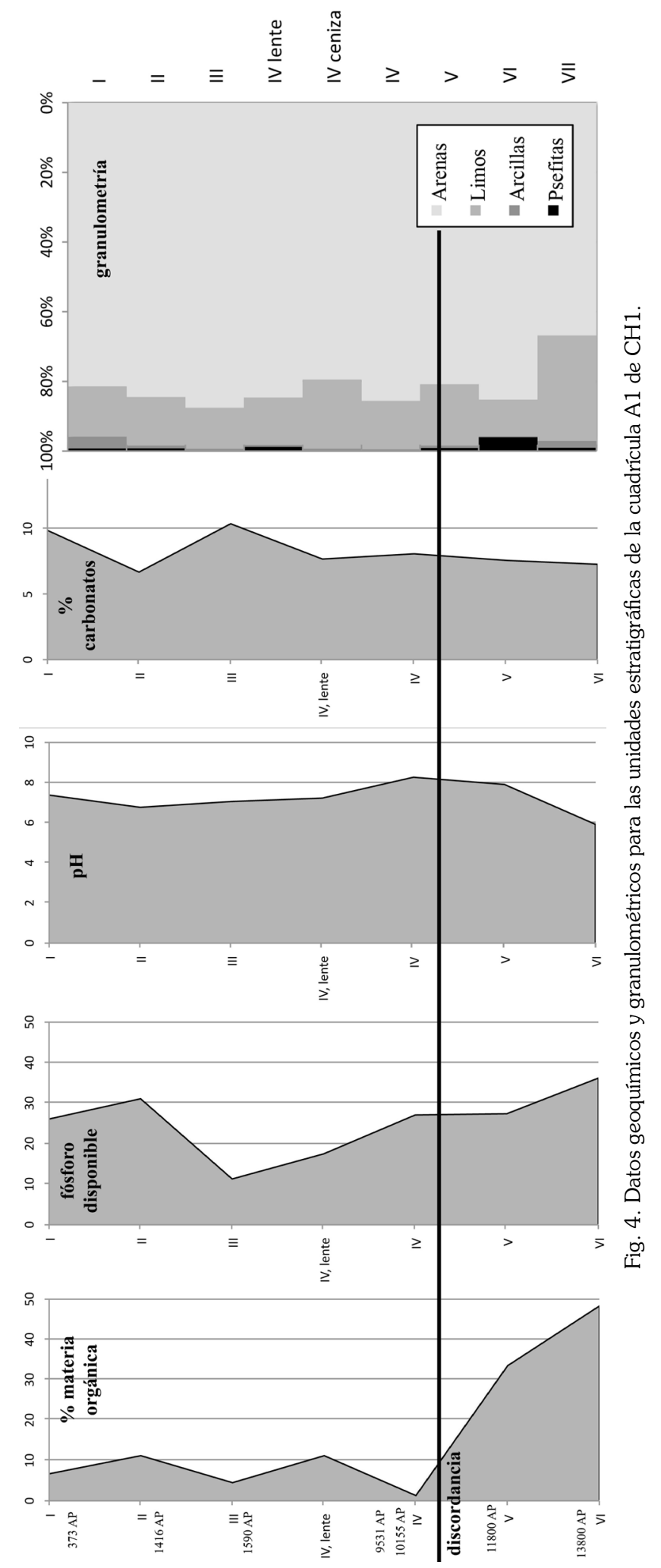

\begin{tabular}{|c|c|c|c|c|c|}
\hline$\supset$ & $\stackrel{0}{+}$ & $\stackrel{\llcorner}{\sim}$ & & & \\
\hline$F$ & $\vec{I}$ & $\begin{array}{l}\stackrel{\sim}{\simeq} \\
\underset{\sim}{N}\end{array}$ & 3 & $\begin{array}{l}N \\
0\end{array}$ & $\stackrel{N}{\sim}$ \\
\hline 2 & $\begin{array}{l}\infty \\
\stackrel{\sim}{\sim}\end{array}$ & $\begin{array}{l}\stackrel{0}{+} \\
+\end{array}$ & $\stackrel{2}{2}$ & $\underset{-}{0}$ & $\stackrel{\infty}{-i}$ \\
\hline 㞬 & $\begin{array}{l}\infty \\
\dot{m}\end{array}$ & $\begin{array}{l}0 \\
\dot{n}\end{array}$ & $\xi$ & $\begin{array}{l}N \\
0\end{array}$ & $\stackrel{m}{m}$ \\
\hline$\tilde{\oplus}$ & 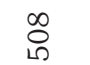 & $\stackrel{\infty}{\alpha}$ & ஸे & $\approx$ & $\stackrel{\infty}{-}$ \\
\hline u & $\stackrel{\infty}{+}$ & $\stackrel{F}{F}$ & 오 & L & ? \\
\hline$\vec{z}$ & $\stackrel{\circ}{-}$ & 0 & ฉె & $\begin{array}{l}0 \\
i\end{array}$ & $\stackrel{\circ}{\dot{m}}$ \\
\hline$\grave{N}$ & $\underset{\sim}{\stackrel{\sim}{\sim}}$ & $\stackrel{\text { ָิ }}{\sim}$ & $\rho$ & ? & ז̊ \\
\hline$\lambda$ & $\stackrel{0}{\sim}$ & $\approx$ & D & $\underset{\sim}{\circ}$ & $\stackrel{\circ}{\circ}$ \\
\hline ஸे & $\stackrel{\text { mे }}{\sim}$ & 芯 & జื & $\stackrel{0}{-}$ & $\Rightarrow$ \\
\hline$\vec{x}$ & $\stackrel{\vec{m}}{\rightarrow}$ & જે & हี & $\begin{array}{l}\infty \\
\infty\end{array}$ & $\Rightarrow$ \\
\hline$\Sigma^{\xi}$ & 옹 & $\underset{\nabla}{\stackrel{\Delta}{\Delta}}$ & $\bar{z}$ & $\hat{\sigma}$ & $\hat{\sigma}$ \\
\hline$\stackrel{*}{*}$ & $\stackrel{\infty}{\stackrel{\infty}{\stackrel{N}{*}}}$ & $\begin{array}{l}\infty \\
\stackrel{\infty}{\infty} \\
\stackrel{n}{n}\end{array}$ & 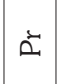 & $\hat{\omega}$ & in \\
\hline 苞 & 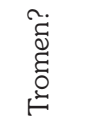 & 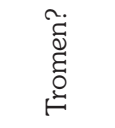 & ن & $\begin{array}{l}N \\
\tilde{N} \\
\tilde{N}\end{array}$ & $\begin{array}{l}\infty \\
\dot{F}\end{array}$ \\
\hline 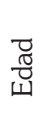 & 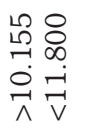 & 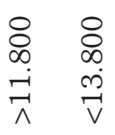 & $\Xi$ & $\stackrel{-}{\hat{N}}$ & $\stackrel{\stackrel{\sim}{N}}{\stackrel{N}{N}}$ \\
\hline ن & $\begin{array}{l}\text { ¿े } \\
\text { के } \\
\text { ठ் }\end{array}$ & $\begin{array}{l}\hat{\tilde{D}} \\
\text { के } \\
\text { రे }\end{array}$ & $\begin{array}{l}0 \\
8 \\
: 7 \\
8 \\
0\end{array}$ & 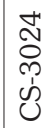 & $\begin{array}{l}\hat{n} \\
\text { के } \\
\text { dे }\end{array}$ \\
\hline 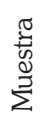 & $\begin{array}{l}\vec{F} \\
\vec{I}\end{array}$ & $\begin{array}{l}\stackrel{N}{\ominus} \\
\vec{U} \\
\stackrel{\vec{U}}{U}\end{array}$ & 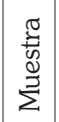 & $\begin{array}{l}\vec{F} \\
\vec{I} \\
\vec{U}\end{array}$ & $\begin{array}{l}\stackrel{N}{\vec{t}} \\
\vec{U} \\
\vec{U}\end{array}$ \\
\hline
\end{tabular}



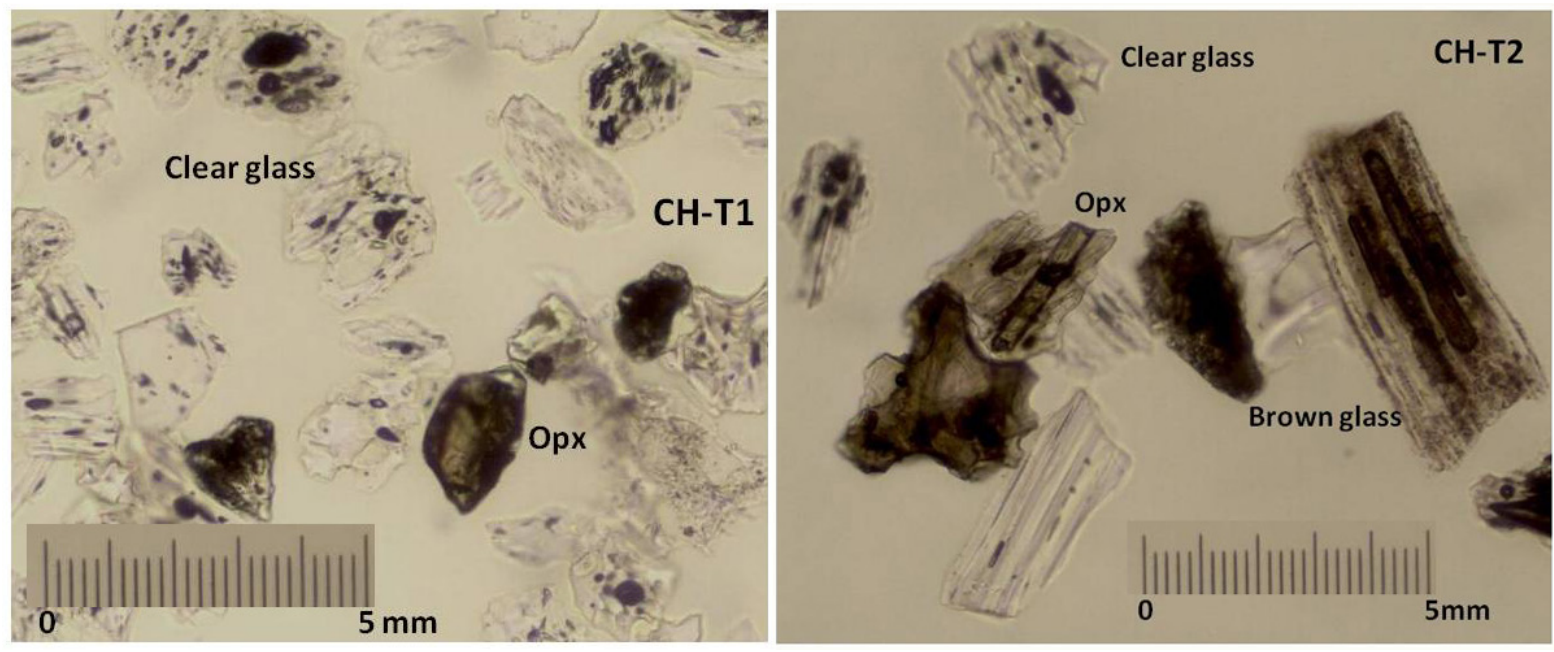

Fig. 5. Imágenes al microscopio de las tefras de CH1 (CH-T1: Tefra 1; CH-T2: Tefra 2).

\section{Tefrocronología}

La Tefra $\mathrm{CH} 1-\mathrm{T} 1$, que es la más reciente, contiene vidrio de tono claro, plagioclasa $y$ ortopiroxeno en baja abundancia (Fig. 5). Tiene un contenido de rubidio $(\mathrm{Rb})$ y lantano $(\mathrm{La})$ más alto que la Tefra $\mathrm{CH} 1-\mathrm{T} 2$, mientras que el contenido de titanio (Ti) y estroncio $(\mathrm{Sr})$ es menor que en esta última (Tabla 2). La Tefra 2, que es la más antigua, presenta vidrio tanto de tono claro como marrón, plagioclasa y ortopiroxeno (Fig. 5). Estos datos implican que estas dos muestras corresponden a tefras diferentes y no a una misma tefra retrabajada en los dos niveles reconocidos a nivel estratigráfico (Fig. 3). La tefra T2 es más máfica y muy probablemente corresponde a una dacita con bajo contenido de $\mathrm{SiO}_{2}$ y un contenido de $\mathrm{FeO}$ mayor que el de la tefra T1. Esta última correspondería composicionalmente a una riodacita o riolita. El volcán Tromen es el más cercano al sitio $\mathrm{CH} 1$ que ha tenido actividad desde fines del Pleistoceno (Folguera et al. 2008) y es, por lo tanto, la fuente más probable para los dos niveles de tefra descriptos. No obstante, dada la ausencia de una secuencia tefrocronológica para esta región, esta inferencia es manejada como una hipótesis de trabajo.

\section{Definición de los componentes estratigráficos}

Sobre la base de la información estratigráfica y cronológica se definieron cuatro componentes sedimentarios y dos hiatos en $\mathrm{CH} 1$ (datos en Barberena, 2014). Aunque la mayor parte de la información se refiere al sector de excavación A1C1-D1, las fechas disponibles para la cuadrícula B1 permiten incluirla en este esquema general (Fig. 6). El componente 1 corresponde al Pleistoceno final y no fue registrado en el sector del sondeo B1. No presenta evidencias arqueológicas in situ y se destaca la notable abundancia de excrementos de carnivoros pequeños con una excelente preservación. Incluye a las unidades VII-V (niveles artificiales 14 a 6), las tefras CH1-T1 y CH1-T2 y tiene una duración aproximada de 3.500 años cal. Se caracteriza por una elevada proporción de materia orgánica y fósforo disponible producto del aporte de los excrementos de megafauna. El límite superior de este componente está dado por una discordancia erosiva denominada hiato 1 , por sobre la cual, unos ca. 3.000 años calendáricos después, se registran las primeras ocupaciones humanas en el sitio. Esta superposición directa, así como la intrusión de estructuras antrópicas holocénicas en sedimentos más antiguos, explica la migración de escasos artefactos líticos a los niveles pleistocénicos. La Tefra 1 apoya sobre esta discordancia y subyace a las evidencias fechadas en el Holoceno temprano.

El componente 2 se ubica inmediatamente por encima de la discordancia e incluye las primeras ocupaciones humanas en $\mathrm{CH} 1$, asignables al Holoceno temprano y con una duración de 2.000 años cal (unidad IV, niveles artificiales 5 y 4). 
OxCal v4.2.3 Bronk Ramsev (2013): r:5 IntCal13 atmospheric curve (Reimer et al. 2013)

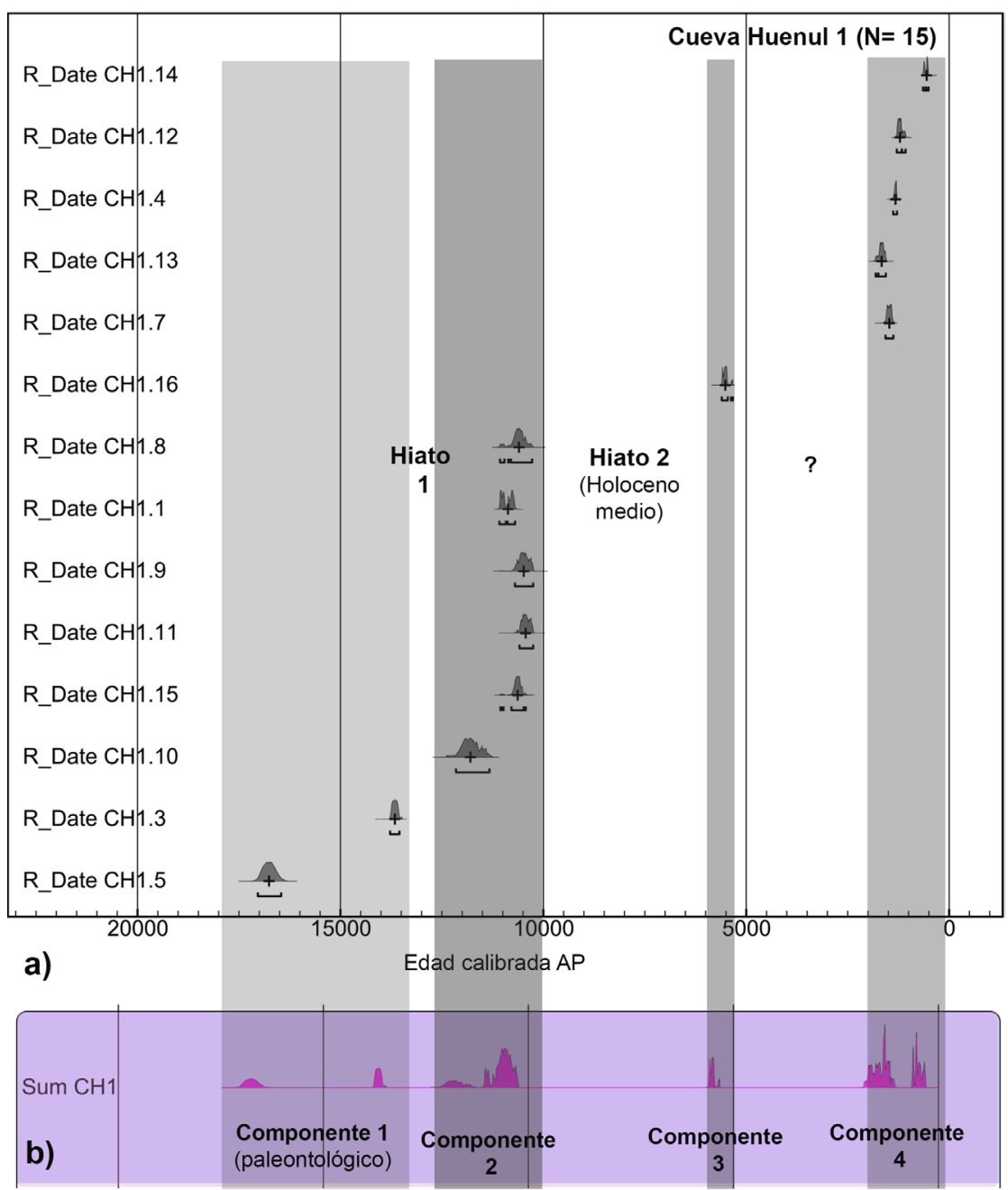

Fig. 6. Distribución de fechas ${ }^{14} \mathrm{C}$ en $\mathrm{CH} 1$ : (A) fechas individuales y definición de componentes; (B) suma de probabilidades de todas las fechas.

Tampoco se registra en el sector B1. Desde un punto de vista cronológico, se discriminan dos pulsos arqueológicos diferentes, lo cual enfatiza el carácter diacrónico en la formación de este componente. A nivel estratigráfico se registra el valor más bajo de materia orgánica en toda la secuencia. Se registró la presencia de al menos dos estructuras de combustión, una de las cuales fue fechada en forma directa. La estructura tipo pozo fechada en 5517 AP define en sí misma al componente 3 , que es la única evidencia del Holoceno medio. Apoyando sobre los niveles del Holoceno temprano, y separado por el hiato 2, que no tiene una expresión material clara, se ubica el componente 4 del Holoceno tardío. Este último componente está representado en los dos sectores de excavación dentro de la cueva, con edades basales de 1.600 (cuadrícula A1) y 1.700 años cal $\mathrm{AP}$ (cuadrícula B1) y una edad más reciente de 400 años AP (en la cuadrícula A1 incluye los niveles artificiales de extracción 3 a 1).

\section{Fauna extinta}

Las unidades que conforman el componente 1 tienen una cronología del Pleistoceno final. El 
principal agente sedimentario es biogénico y se vincula a la acción de especies extintas mediante el aporte de excrementos en gran abundancia, en conjunto con la sedimentación eólica (Barberena, 2014). En todo el sedimento excavado correspondiente a este período, sólo se recuperó un espécimen óseo con potencial diagnóstico, el cual procede de la cuadrícula C1-D1. Dadas las excelentes condiciones de preservación registradas, esto sugiere una muy baja tasa de depositación de materiales óseos en relación con la intensa sedimentación biótica registrada. El material óseo es un hioides completo que se asigna a un taxón correspondiente al Orden Pilosa. En la Figura 7 se compara este elemento con uno de Scelidotherium leptocephalum procedente de las colecciones del Museo de Ciencias Naturales de La Plata (MLP). Este registro se ubica entre las fechas de 16.759 y 13.658 AP.

El basihial y los tirohiales están fusionados formando un único hueso impar, el basi-tirohial. Dicha condición se ha mencionado para los Xenarthra y, entre éstos, el de CH1 se asemeja a los Pilosa en los que no hay un proceso lingual (Pérez et al. 2010). El tamaño del ejemplar de CH1 resulta similar a algunos especímenes asignados a Scelidotherium (Pérez et al. 2010), sin embargo, la mayoría de los basi-tirohiales de los perezosos descriptos del Cuaternario son de talla algo mayor.

El basi-tirohial de $\mathrm{CH} 1$ es parabólico (el ancho es mayor que la longitud; Fig. 7A,C) y tiene forma de "U" en vista dorsal, similar a Glossotherium y Paramylodon (Pérez et al. 2010). En Scelidotherium, el hueso tiene forma de "V" (Fig. 7B,D). El basi-tirohial de $\mathrm{CH} 1$ conserva solo la faceta derecha para la articulación con el ceratohial. Esta es pequeña, oval y con el eje mayor orientado horizontalmente. En los milodóntidos conocidos, las facetas del ceratohial están mejor definidas que en $\mathrm{CH} 1$; aunque tanto $\mathrm{CH} 1$ como los otros milodóntidos difieren claramente de Megatherium por presentar este último facetas prominentes y proyecciones anteriores del hueso (Pérez et al. 2010). Las astas mayores están levemente comprimidas lateralmente. Las facetas para el cartílago tiroides en $\mathrm{CH} 1$ son circulares y se ubican en la cara posterior de las astas mayores (Fig. 7E). En Scelidotherium, las facetas son ovales y oblicuas, orientadas postero-ventralmente (Fig. 7F;
MLP 3-671, MACN-PV 18118) o ventralmente (MACN-PV 13649). En vista lateral (Fig. 7D), el asta mayor de $\mathrm{CH} 1$ tiene un proceso ubicado sobre el borde dorsal y cerca del medio de su longitud; el borde ventral es cóncavo. En Scelidotherium, el proceso dorsal es más pequeño. Variaciones en la forma del borde ventral fueron detectadas entre los especímenes comparados de Scelidotherium. Es cóncavo anteriormente y casi recto posteriormente en MLP 3-671 (Fig. 7F) y MACN-PV 18118, mientras que posee una cresta ventral en MACNPV 13649.

Variaciones intraespecíficas parecen ser importantes en el hueso hioides. Estas fueron reportadas para humanos $e$ incluyen tanto diferencias en la longitud de los cuernos mayores como el grado de fusión del basihial y tirohial (Miller et al. 1998). Considerando la morfología del ejemplar de $\mathrm{CH} 1$ y su similitud con el basitirohial de los pilosos (excepto Megatherium), se asigna tentativamente este material a un perezoso terrestre. Análisis futuros con una muestra mayor permitirán eventualmente precisar esta determinación.

\section{Zooarqueología}

Los restos de micro-fauna han sido estudiados desde un punto de vista tafonómico y zooarqueológico, indicando que el conjunto de $\mathrm{CH} 1$ fue generado por la actividad depredadora de aves Strigiformes, principalmente Tyto alba (Fernández et al. 2012). En estos materiales se observó una muy buena preservación producto de una rápida depositación en la matriz sedimentaria, aunque con ciertas evidencias de pisoteo. Estos datos tafonómicos son clave para evaluar la amplitud dietaria de las sociedades humanas del pasado. Por otra parte, la secuencia de micromamíferos reveló una diversidad taxonómica relativamente constante a través de la secuencia, la cual fue relacionada con una estabilidad ambiental, aunque con una mayor heterogeneidad durante el Holoceno, incluyendo un mosaico de estepas arbustivas, peladales y áreas rocosas abiertas (Fernández et al. 2011, 2012).

En la Tabla 3 se presentan los resultados del análisis faunístico de la cuadrícula $\mathrm{A} 1$. Se recuperó un total de 1517 especímenes óseos, de los cuales se pudo determinar taxonómicamente un $39,4 \%$. 

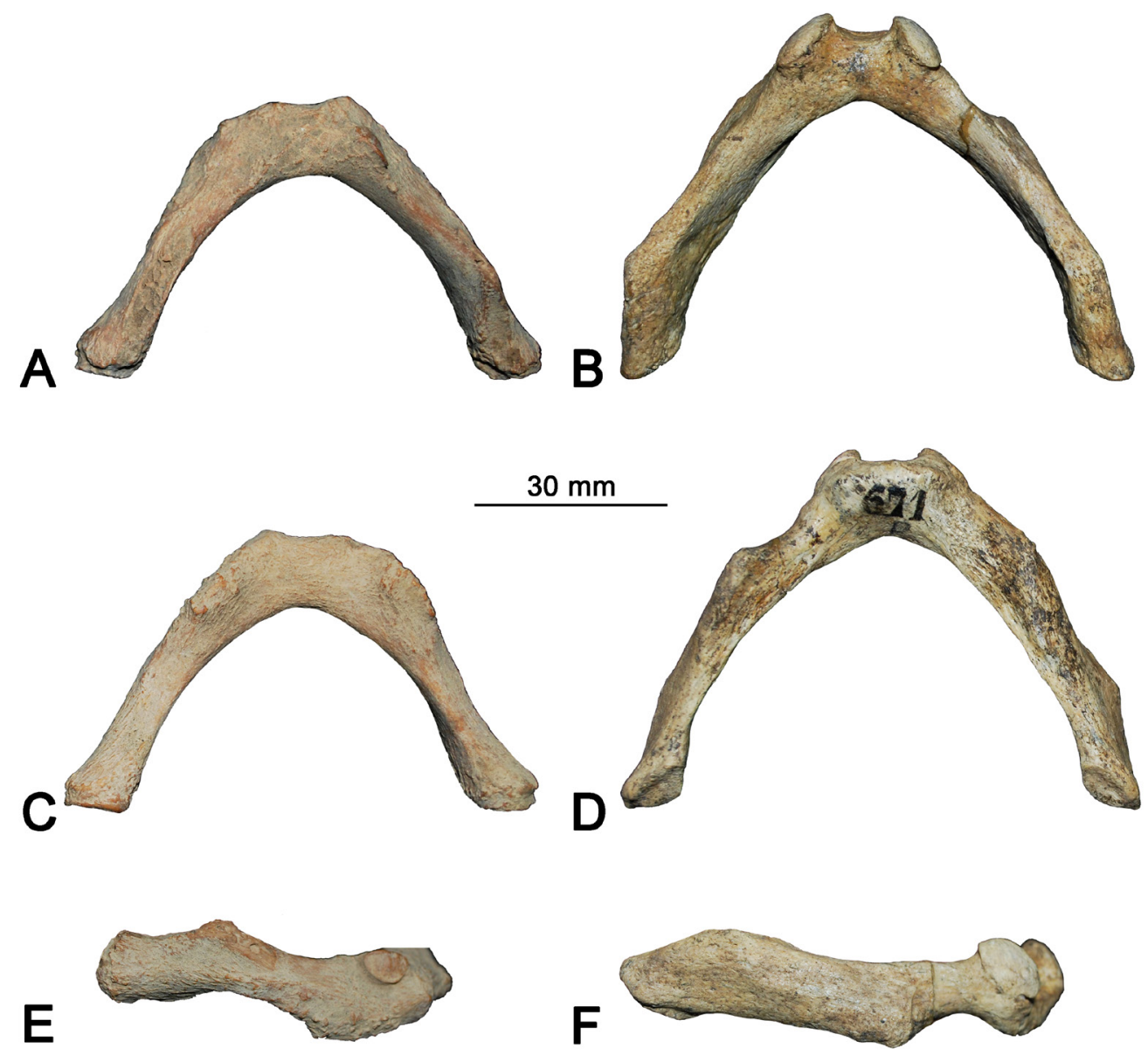

Fig. 7. Hueso basi-tiroial (hueso en V) de $\mathrm{CH} 1$ a la izquierda y Scelidotherium leptocephalum (MLP 3-671) a la derecha en vista dorsal (A,B), ventral (C,D) y lateral (E,F).

El taxón más abundante entre los identificados es Lama guanicoe, con $13 \%$ del total. Cabe señalar que $23,7 \%$ corresponde a restos no determinados de Mammalia, una parte del cual puede corresponder a especímenes de L. guanicoe que han perdido sus zonas diagnósticas. El resto de los taxa identificados (Rhea pennata, Dasypodidae, Carnivora, Lagidium viscacia, etc.) se encuentra representado en muy bajas proporciones.

Por razones de espacio no se presenta un estudio formacional exhaustivo, sino un perfil general de la muestra (Tabla 4). El mismo sugiere una excelente preservación de los materiales con valores virtualmente nulos de meteorización por exposición a condiciones subaéreas (sensu Behrensmeyer, 1978) y con la presencia de tejidos blandos en muchos especímenes de cronología diversa. Tampoco se registra incidencia de marcas de raíces. Ambos indicadores son coherentes con la ubicación de la cuadrícula A1, en el sector profundo de la cueva y con un enterramiento relativamente rápido de los materiales, debido en gran parte a la acción de los humanos como agente sedimentario Hay una frecuencia mínima de marcas de roedor $(0,7 \%)$ y carnívoros $(0,3 \%)$ sobre los huesos, aunque hay otros indicadores de la presencia de estos agentes: se detectaron sedimentos removidos por la acción de animales fosoriales, en particular en el contacto entre las unidades del Holoceno temprano y las del Pleistoceno tardío, así como también abundantes coprolitos de carnívoro y roedor (Beltrame et al. 2011). Las huellas antrópicas se registran en un 5,9\% del conjunto total, aunque con diferencias entre los 


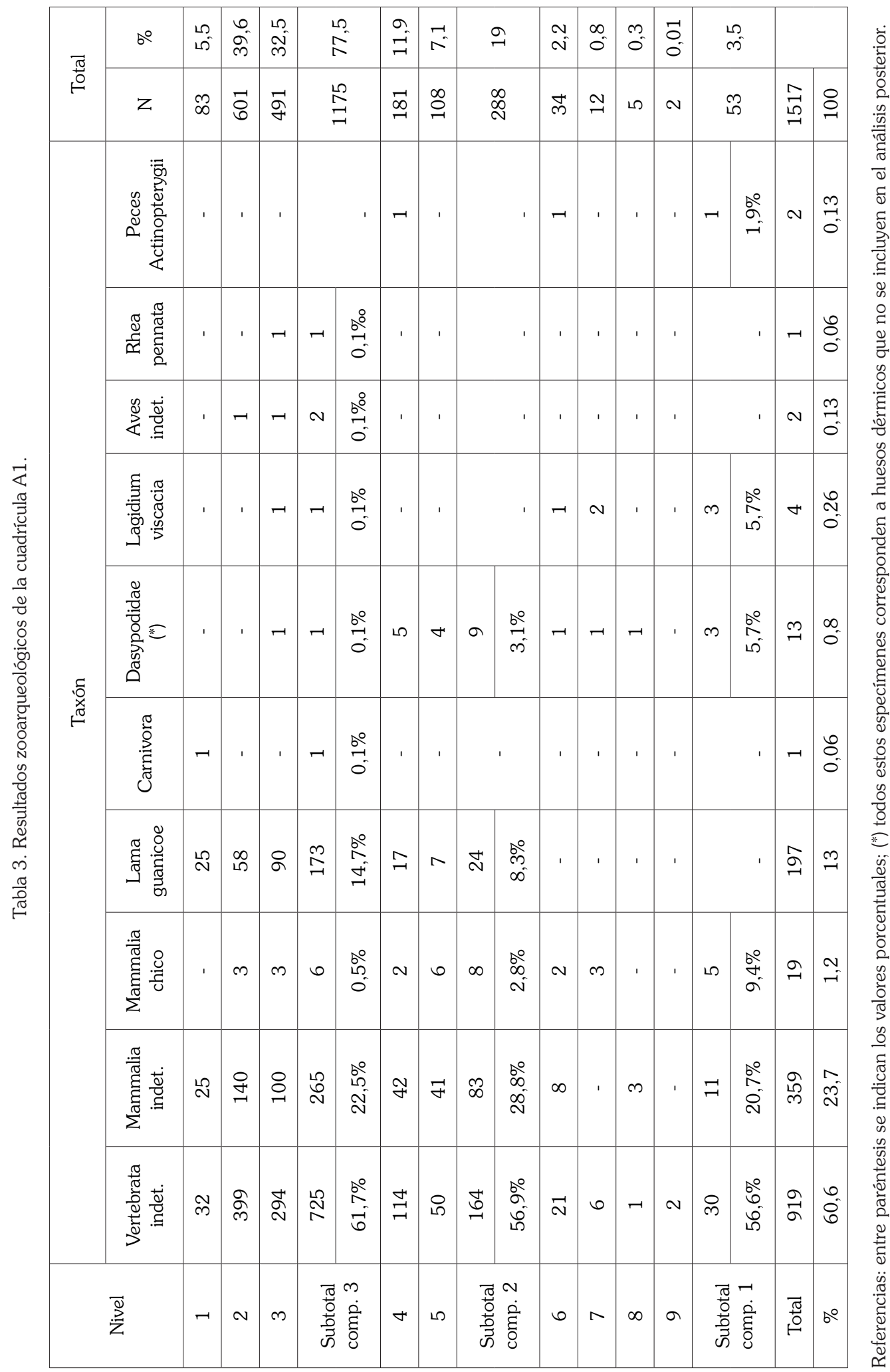


Tabla 4. Evidencias tafonómicas y antrópicas en los conjuntos faunísticos de la cuadrícula A1.

\begin{tabular}{|c|c|c|c|c|c|}
\hline Nivel & Manganeso & $\begin{array}{l}\text { Quemado- } \\
\text { calcinado }\end{array}$ & $\begin{array}{l}\text { Marcas } \\
\text { roedor }\end{array}$ & $\begin{array}{l}\text { Marcas } \\
\text { carnívoro }\end{array}$ & $\begin{array}{c}\text { Huellas } \\
\text { antrópicas (+) }\end{array}$ \\
\hline 1 & - & 8 & - & 2 & 9 \\
\hline 2 & - & 90 & 6 & 2 & 23 \\
\hline 3 & - & 22 & 2 & - & 26 \\
\hline $\begin{array}{l}\text { Subtotal } \\
\text { Comp. } 4\end{array}$ & - & 120 & 8 & 4 & 58 \\
\hline$\%$ & 0 & 10,2 & 0,7 & 0,4 & 4,9 \\
\hline 4 & - & 27 & 2 & - & 26 \\
\hline 5 & - & 1 & - & - & 5 \\
\hline $\begin{array}{l}\text { Subtotal } \\
\text { Comp. } 2\end{array}$ & - & 28 & 2 & - & 31 \\
\hline$\%$ & 0 & 9,8 & 0,7 & 0 & 10,8 \\
\hline 6 & - & 4 & - & 1 & - \\
\hline 7 & - & 1 & - & - & - \\
\hline 8 & - & - & - & - & - \\
\hline 9 & - & - & - & - & - \\
\hline $\begin{array}{l}\text { Subtotal } \\
\text { Comp. } 1\end{array}$ & - & 5 & - & 1 & - \\
\hline$\%$ & 0 & 9,4 & 0 & 1,9 & 0 \\
\hline Total general & - & 153 & 10 & 5 & 89 \\
\hline$\%$ & 0 & 10,1 & 0,7 & 0,3 & 5,9 \\
\hline
\end{tabular}

Referencias: (+) incluye huellas de corte, lascado, lascas óseas y machacado.

tres componentes. El componente 1 no presenta huellas, mientras que el del Holoceno temprano duplica en su proporción al del Holoceno tardío (10,8 vs. $4,9 \%)$. Esto sugiere que los conjuntos tempranos evidencian una mayor intensidad de procesamiento de las carcasas que los tardíos. Un $10 \%$ del conjunto total presenta evidencias de acción del fuego (quemado, calcinado) en forma semejante en los tres componentes. En el caso del componente 1, para el que se contempla una génesis natural, cabe pensar la incidencia de migración vertical. Dada la escasa separación vertical entre los componentes arqueológicos 2 y 4 no se puede descartar que, en algunos casos, la acción del fuego haya ocurrido en forma posterior a la depositación (Bennett 1999).
Integrando todos los indicadores tafonómicos y arqueológicos aquí descriptos planteamos que la depositación de los conjuntos faunísticos de la cuadrícula A1 responde principalmente a la conducta humana. Defendemos también que los procesos de destrucción post-depositacional tienen una baja incidencia en los componentes 2 y 4 , ocasionando pérdida del valor diagnóstico en algunos casos pero una baja destrucción. El componente 1 presenta condiciones de formación diferentes que sugieren una mayor importancia de estos procesos.

Sobre la base de la Tabla 3, en la Figura 8 se comparan las frecuencias de los principales taxa representados en los tres componentes analíticos. No se incluyen los taxa peces, $R$. pennata y 


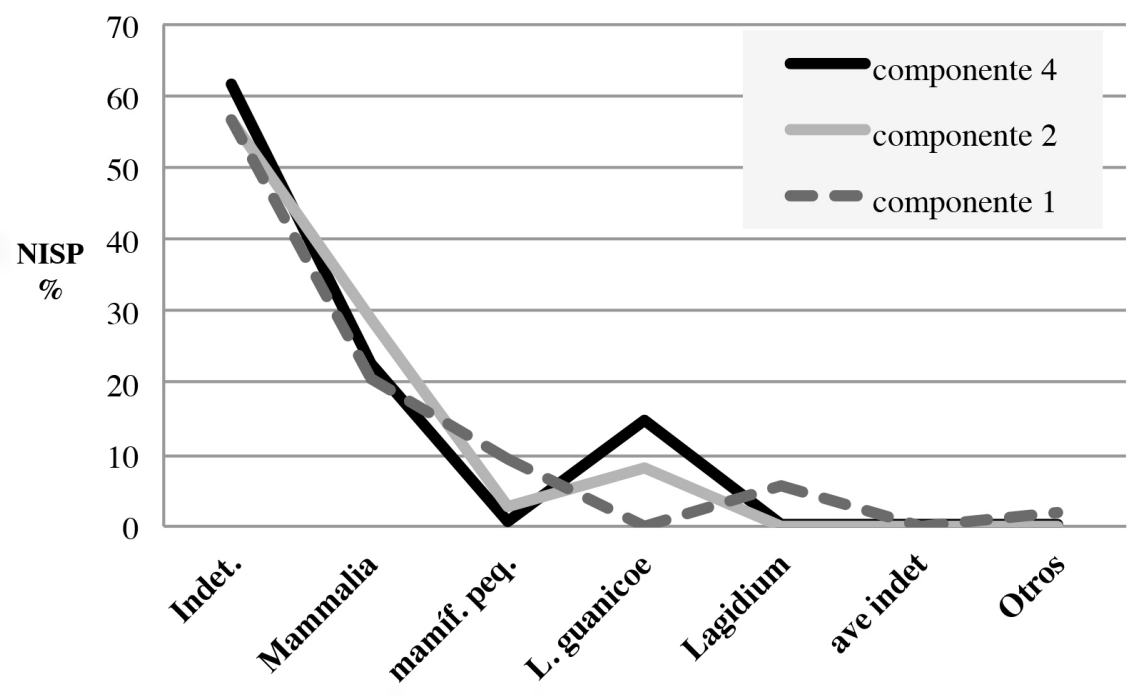

Fig. 8. Proporciones taxonómicas en la fauna de la cuadrícula A1.

Carnivora que representan respectivamente $0,1 \%$ del total, así como tampoco la micro-fauna que, como se mencionó, responde principalmente a la acción de aves rapaces (Fernández et al. 2012). En una primera instancia nos enfocamos en la comparación de los componentes 2 (11.798$10.483 \mathrm{AP})$ y $4(\leq 1.500 \mathrm{AP})$.

No se observan importantes diferencias en la composición de los componentes 2 y 4. El componente 2 tiene una representación levemente mayor del taxón Mammalia que el 3 (28,8 vs. $22,5 \%)$ mientras que, por el contrario, es menor en el caso de L. guanicoe (8,3 us. $14,7 \%)$. Estas diferencias son equivalentes en torno a un $6 \%$ de los respectivos subtotales y pueden responder básicamente a procesos post-depositacionales de destrucción de baja intensidad, que disminuyen el valor diagnóstico de los especímenes en el conjunto más antiguo. Excluyendo los especímenes de huesos dérmicos de Dasypodidae, se registró una mayor abundancia de especímenes asignados a 'Mammalia chico' en el componente 2 vs. el 4 (2,8 vs. 0,5\%). Aunque de muy pequeña magnitud, esta diferencia puede ir en la línea de los registros del Holoceno temprano publicados para los sitios Cueva Traful I (Crivelli Montero et al. 1993; Cordero 2011) y Cueva Epullán Grande (Crivelli Montero et al. 1996; Cordero, 2009), ubicados en el sur de la provincia del Neuquén. L. guanicoe es el recurso faunístico clave en los componentes 2 y 4 . En este sentido, al separar los aportes naturales, todos los conjuntos de esta cuadrícula se caracterizan por una baja diversidad taxonómica. Esto es relevante en el marco de debates sobre amplitud de dieta en escala regional y macro-regional (Neme \& Gil 2008b; Cordero 2011).

\section{Arqueobotánica}

El registro arqueobotánico de $\mathrm{CH} 1$ refleja el aprovechamiento de recursos vegetales autóctonos, tales como los géneros Prosopis, Schinus, Retanilla y Maihueniopsis (Tabla 5). Los restos hallados evidencian señales tafonómicas de procesamiento antrópico, ya que los endocarpos se encuentran fragmentados y con señales de fricción a lo largo del segmento holocénico de la secuencia. Esto sugiere que al menos una parte de estas plantas fue procesada antes de ser utilizadas. El taxón más frecuente es Prosopis sp. El registro recurrente de las partes del fruto y/o sus semillas puede ser asignado al consumo alimenticio, lo cual concuerda con la información brindada por numerosas fuentes etnohistóricas y etnobotánicas (Ladio \& Lozada, 2009; Capparelli \& Lema, 2011). Cabe señalar que el registro arqueobotánico evidencia edades tempranas considerando las fechas obtenidas para un fruto de Retanilla patagonica (10.436 AP) y Prosopis sp. (10.633 AP). Todos los taxones encontrados son característicos de ambientes áridos y poseen uno o más usos documentados. 
Tabla 5. Síntesis del registro arqueobotánico en la cuadrícula A1 de CH1.

\begin{tabular}{|c|c|c|}
\hline Taxón & Frecuencia & Parte/s \\
\hline Atriplex lampa & 1 & bracteola \\
\hline Eruca vesicaria & 1 & fruto \\
\hline Chuquiraga erinacea & 1 & pedúnculo floral \\
\hline Larrea divaricata & 8 & hojas y mericarpos \\
\hline Lagenaria sp. & 3 & fragmentos de cáscara \\
\hline Maihuenopsis darwinii & 10 & frutos y espinas \\
\hline Prosopis sp. & 274 & vainas, semillas y endocarpos \\
\hline Retanilla patagonica & 1 & fruto \\
\hline Schinus polygamus & 16 & endocarpo \\
\hline Total & 315 & \\
\hline
\end{tabular}

La presencia de macrorrestos del taxón doméstico Lagenaria sp. indica similitudes con el registro de contextos en el sur de Mendoza (Llano, 2011) y el centro-oeste de Neuquén (Fernández, 1988-1990) durante el Holoceno tardío. En general Lagenaria ha sido utilizada para la confección de contenedores, instrumentos musicales y flotadores de pesca, dada la característica de sus frutos de cáscara dura y boyante (Erickson et al. 2005), y en menor proporción como fuente de alimento. Uno de los fragmentos hallados en $\mathrm{CH} 1$ presenta una pequeña evidencia de pirograbado, respaldando una interpretación artefactual (Tabla 5).

\section{Representaciones rupestres}

El presente relevamiento se inició en 2012. Aquí se resumen las primeras tendencias observadas cuyo estudio será profundizado en futuros trabajos. En primer lugar, cabe destacar que el arte rupestre de $\mathrm{CH} 1$ está conformado en su totalidad por pinturas, las cuales muestran distinto estado de preservación resultado de la acción de la humedad y de las acreciones minerales. Los motivos se encuentran distribuidos en 11 paneles de distinta extensión, diferenciados a partir de la presencia de fracturas, cambios en la orientación de la roca soporte y sectores de pared sin pinturas. Hasta el momento se han identificado más de 150 motivos, tanto simples como compuestos, lo cual demuestra la gran importancia de este sitio.

Entre las representaciones documentadas se observó el amplio predominio de los motivos abstractos (más del 70\%) por sobre los figurativos (Fig. 9). Dentro de los primeros se identificaron formas geométricas simples como círculos, líneas y puntos, así como figuras almenadas, escalonadas y de simetría axial, gran variedad de líneas paralelas y diversos tipos de encadenados. Se destacan algunos motivos de factura más compleja o que presentan mayores dimensiones como casos de cruciformes de $1 \mathrm{~m}$ de alto. A pesar de presentarse en baja frecuencia, entre los motivos figurativos se distinguen zoomorfos y antropomorfos. Entre los primeros se diferenciaron dos tipos, ñandú y cuadrúpedo indeterminado, mientras que el único tipo antropomorfo documentado es la figura humana. A la amplia variedad de tipos de motivos definidos se agrega una gran cantidad de motivos indeterminados por deterioro.

Por otra parte, se observa el predominio de motivos monocromos ejecutados en distintos tonos de rojo, si bien se registran algunos casos de bicromía (Fig. 9). Otras tonalidades registradas en menor frecuencia incluyen amarillo, blanco y negro. En forma preliminar, se propone que la mayor parte de las pinturas de $\mathrm{CH} 1$ pueden ser asignadas al Holoceno tardío y, más específicamente a los últimos 1.600 años, tal como se sugiriera para los demás sitios del área de estudio (Romero \& Re, 2014). Esta observación se apoya, por un lado, en los hallazgos previamente comentados del componente 4 y el marco cronológico provisto por los fechados disponibles, así como también en la similitud morfológica existente entre las pinturas consideradas y otros motivos registrados 
en áreas cercanas que fueron asignados por otros investigadores a momentos tardíos -entre los siglos VIII y XV de nuestra era- (ver citas en Gradin 1997-1998). No obstante, interesa destacar que se documentaron 23 casos de superposiciones que involucran una amplia variedad de tipos de motivos, así como diferentes tonalidades, las cuales permitirán evaluar a futuro los distintos momentos de ejecución de las pinturas.

\section{Tecnología lítica}

Se recuperaron 1450 artefactos en la cuadrícula A1 (Tablas 6 y 7). Los componentes
2 y 4 (Holoceno temprano y tardío) registraron la mayor frecuencia de materiales líticos $(23,4$ y $75,2 \%$, respectivamente), siendo marginal $(1,4 \%)$ el aporte del componente 1 (Pleistoceno final).

Los artefactos han sido mayormente confeccionados sobre obsidiana $(85,2 \%)$, que predomina a lo largo de toda la secuencia (Tabla 6). Esta materia prima es de disponibilidad local (fuente Cerro Huenul, Durán et al. 2004; Barberena et al. 2011). El análisis geoquímico de muestras naturales locales y artefactos arqueológicos ha mostrado que la obsidiana Huenul es la más frecuente en la muestra de $\mathrm{CH} 1$ (Barberena et al. 2011), aunque recientemente se registró la presencia de obsidiana
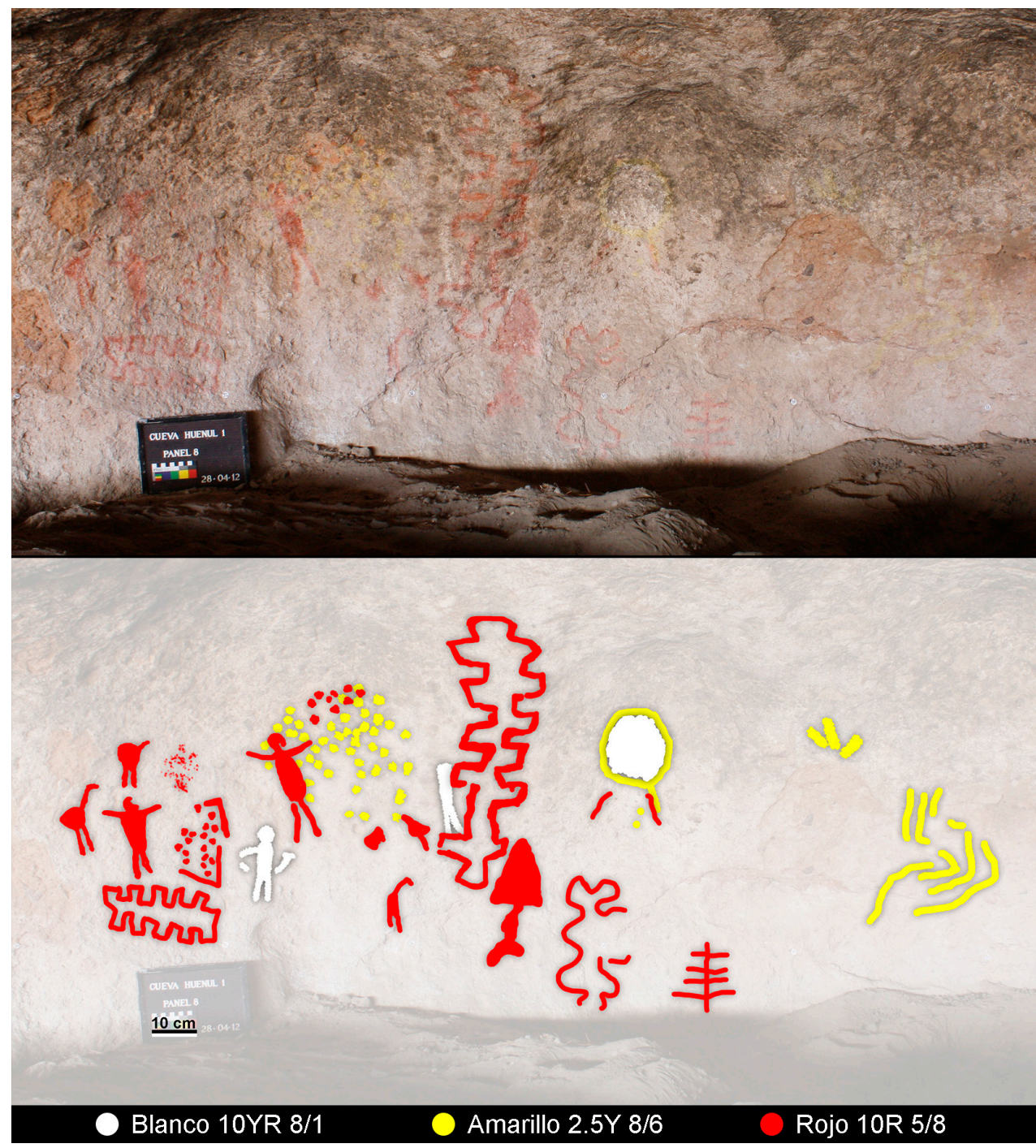

Fig. 9. Foto y calco de los motivos registrados en el Panel 8. 
de la fuente Laguna del Maule (datos inéditos). En el componente 2, la obsidiana es seguida en frecuencia por basalto, sílice y calcedonia y en el componente 4, por calcedonia, silice y rocas indeterminadas. Si bien la obsidiana es la materia prima predominante en la secuencia, para los momentos tardíos se registra un incremento en el aporte de la calcedonia. Por otra parte, el basalto presenta su mayor frecuencia en los niveles correspondientes al componente 2 .

Con respecto a las clases artefactuales, la más representada es la correspondiente a microdesechos, que incluyen aquí los desechos de tamaño $\leq 5 \mathrm{~mm}^{2}$ (grilla de tamaños sensu Franco, 2002) y constituyen el $69,9 \%$ de la muestra. También son frecuentes los desechos (>5 mm), los cuales representan el $29 \%$ del total. En el componente 1 , que incluye restos paleontológicos, los desechos son la clase más representada (95\%). Como una medida conservadora, por el momento, los artefactos recuperados en los niveles asignados al Pleistonceno final son considerados materiales redepositados, por lo que son excluidos de la discusión tecnológica a nivel temporal. La información disponible sugiere la existencia de procesos de migración vertical en esos niveles, probablemente favorecidos por el pisoteo de los distintos agentes en la cueva, tal como lo evidencian los restos de micro-fauna. El origen de estos materiales será evaluado en el futuro.

En los componentes 2 y 4 se observa un predominio de lascas angulares (28,93\%), lascas de reactivación/formatización de filos $(14,96 \%)$ y por último lascas primarias (8,98\%). El 71,32\% de los desechos de ambos componentes no presenta reserva de corteza. Los tamaños más frecuentes corresponden a los desechos más pequeños (10 $\mathrm{mm})$, representando el $64,8 \%$ del total de ambos componentes. En menor frecuencia aparecen los instrumentos y núcleos. Los instrumentos $(\mathrm{N}=8$; $0,5 \%$ de la muestra) sólo fueron recuperados en el componente 4, correspondiente al Holoceno tardío. Los grupos tipológicos más representados son las lascas con rastros complementarios $(\mathrm{N}=3)$ y puntas de proyectil $(\mathrm{N}=2)$. En menor medida, se registraron otros grupos tipológicos: un chopping tool, un RBO y un instrumento indeterminado. Una de las puntas de proyectil, que presenta impregnaciones de ocre, está confeccionada sobre obsidiana procedente de la fuente Laguna del Maule (datos inéditos). La otra fue manufacturada sobre calcedonia, siendo éste el único instrumento no manufacturado sobre obsidiana. Predominan los instrumentos con $25 \%$ de corteza $(62,5 \%)$. Los

Tabla 6. Frecuencia de materias primas líticas por nivel de la cuadrícula A1.

\begin{tabular}{|c|c|c|c|c|c|c|c|}
\hline Nivel & Componente & obsidiana & calcedonia & silice & basalto & indeterminado & Total \\
\hline 2 & \multirow{2}{*}{$\begin{array}{c}4 \\
\text { (Holoceno } \\
\text { tardío) }\end{array}$} & $246(76,88)$ & $72(22,5)$ & $1(0,31)$ & - & $1(0,31)$ & $320(22,07)$ \\
\hline 3 & & $667(86,51)$ & $100(12,97)$ & $3(0,39)$ & - & $1(0,13)$ & $771(53,17)$ \\
\hline 4 & \multirow{2}{*}{$\begin{array}{c}2 \\
\text { (Holoceno } \\
\text { temprano) }\end{array}$} & $218(90,83)$ & $2(0,83)$ & $8(3,34)$ & $12(5)$ & - & $240(16,55)$ \\
\hline 5 & & 89 (89) & $1(1)$ & $4(4)$ & $6(6)$ & - & $100(6,90)$ \\
\hline 6 & \multirow{4}{*}{$\begin{array}{l}1 \\
\text { eistoceno } \\
\text { final) }\end{array}$} & $7(100)$ & - & - & - & - & $7(0,48)$ \\
\hline 7 & & $7(70)$ & - & $1(10)$ & $2(20)$ & - & $10(0,69)$ \\
\hline 8 & & $1(50)$ & - & $1(50)$ & - & - & $2(0,14)$ \\
\hline 9 & & - & - & - & - & - & - \\
\hline Total & & 1235 & 175 & 18 & 20 & 2 & $1450(100)$ \\
\hline
\end{tabular}

Referencias: entre paréntesis se indican los valores porcentuales.

2 La distinción operativa de desechos y microdesechos responde a la posibilidad de diferenciar macroscópicamente los atributos morfológicos (talón, estado, clase de lasca) de los desechos con tamaños superiores a $5 \mathrm{~mm}$ y no así en aquellos de dimensiones inferiores. 
tamaños más representados están comprendidos entre 30 y 35 mm (el 50\% de los instrumentos posee $35 \mathrm{~mm}$ de tamaño, y el $25 \%, 30 \mathrm{~mm}$ ).

Se recuperaron ocho núcleos $(0,5 \%$ de la muestra): uno de lascados aislados del componente 2 y seis del componente 4 (cuatro de lascados aislados y dos bipolares). El núcleo del componente 2 tiene un tamaño de $15 \mathrm{~mm}$ mientras que aquellos del componente 4 están comprendidos entre 25 y $40 \mathrm{~mm}$. En el componente 1 se recuperó un único núcleo bipolar de tamaño superior $(55 \mathrm{~mm}$ ) a los registrados en los componentes holocénicos.

El predominio de artefactos sin reserva de corteza sugiere un énfasis en la representación de estadios intermedios y finales de manufactura. La reactivación $y / o$ formatización de instrumentos es una actividad tecnológica bien registrada en el sitio, en el Holoceno temprano y tardío (Tabla 8). Esto queda evidenciado por la elevada frecuencia de lascas de reactivación/formatización en los componentes 2 y 4 (12,03 y 16,42\% del total de desechos, respectivamente). Por otra parte, la presencia de lascas vinculadas con los primeros estadios de talla (primarias, secundarias y con dorso natural) en ambos componentes muestra la realización de actividades de desbaste inicial de nódulos o explotación de núcleos que aún conservaban corteza. Esto último, sumado a la información geoquímica ya disponible y al hecho de que $\mathrm{CH} 1$ se emplaza en la fuente de Cerro Huenul, sugiere la explotación de nódulos de obsidiana disponibles en la inmediata vecindad. Este uso de la fuente se manifiesta en el alto porcentaje de esta materia prima y en el aporte de artefactos con reserva de corteza $(27,75 \%$, excluyendo los microdesechos). Para el resto de las litologías, el porcentaje de artefactos con reserva de corteza es de sólo el 4,13\%. Esto sugiere el énfasis en la realización de estadios finales de talla en esas materias primas.

La baja frecuencia general de instrumentos en toda la secuencia sugiere que su descarte habría tenido lugar en otros emplazamientos. Adicionalmente, a excepción de las puntas de proyectil, se observa el predominio de instrumentos informales (sensu Andrefsky, 1994; cf. Escola, 2004) con filos naturales o poco formatizados y escasas evidencias de reactivación. Esto marca un fuerte contraste entre los instrumentos recuperados en el sitio y aquellos que, por la información obtenida a partir del estudio de los desechos, habrían sido formatizados y/o mantenidos allí.

\section{Tecnología cerámica}

El material cerámico en $\mathrm{CH} 1$ es sumamente escaso, habiéndose recuperado tres tiestos en superficie y dos en estratigrafía, en la cuadrícula B1. Los tres fragmentos hallados en superficie poseen espesores de entre 6 y 7,5 mm, sin tratamiento de superficie y con antiplásticos que alcanzan hasta $2 \mathrm{~mm}$ de tamaño máximo. Estas características

Tabla 7. Composición tecno-tipológica del conjunto lítico recuperado en la cuadrícula A1 de CH1.

\begin{tabular}{|c|c|c|c|c|c|c|}
\hline Nivel & Componente & microdesechos & desechos & instrumentos & núcleos & Total \\
\hline 2 & \multirow{2}{*}{$\begin{array}{c}4 \\
\text { (Holoceno tardío) }\end{array}$} & $214(66,88)$ & $100(31,25)$ & $5(1,56)$ & $1(0,31)$ & $320(22,07)$ \\
\hline 3 & & $594(77,14)$ & $168(21,82)$ & $3(0,39)$ & $5(0,65)$ & $770(53,10)$ \\
\hline 4 & \multirow{2}{*}{$\begin{array}{c}2 \\
\text { (Holoceno } \\
\text { temprano) }\end{array}$} & $143(59,58)$ & $97(40,42)$ & - & - & $240(16,55)$ \\
\hline 5 & & $63(63)$ & $36(36)$ & - & $1(1)$ & $100(6,90)$ \\
\hline 6 & \multirow{4}{*}{$\begin{array}{c}1 \\
\text { (Pleistoceno final) }\end{array}$} & - & $7(87,5)$ & - & $1(12,5)$ & $8(0,55)$ \\
\hline 7 & & - & $10(100)$ & - & - & $10(0,69)$ \\
\hline 8 & & - & $2(100)$ & - & - & $2(0,14)$ \\
\hline 9 & & - & - & - & - & - \\
\hline Total & & 1014 & 420 & 8 & 8 & $1450(100)$ \\
\hline
\end{tabular}

Referencias: entre paréntesis se indican los valores porcentuales. 
macroscópicas coinciden con la mayor parte del registro en escala regional, y se corresponden a nivel general con las decisiones de manufactura que se pueden considerar de 'baja inversión tecnológica' (sensu Simms et al. 1997). En forma preliminar se considera a este subconjunto como de manufactura local, aunque esto debe ser evaluado en función de análisis petrográficos y geoquímicos (Chidiak, 2013).

Los dos fragmentos recuperados en estratigrafía proceden del nivel $6(60-70 \mathrm{~cm})$ de la cuadrícula B1. Esta cuadrícula presenta una fecha de $1.269 \pm 46$ años AP para el nivel artificial 2 y otra de $1.753 \pm 47$ para el nivel 6 (Tabla 1), del cual procede la cerámica. Aunque se ha verificado importante migración de material en $\mathrm{B} 1$, es defendible que las muestras subyacen a la primera fecha y tienen una edad mayor. Las evidencias de la cuadrícula B1 en su conjunto se asocian al componente 4. A nivel macroscópico, se observa una importante diferencia entre estos dos tiestos y aquellos recuperados en superficie. Los tiestos procedentes de estratigrafía tienen espesores de 4 y $5 \mathrm{~mm}$, con superficies bruñidas negra y roja respectivamente. En ambos casos, el tamaño máximo de los antiplásticos es de 0,5 mm. Se considera que estos fragmentos evidencian una 'elevada inversión tecnológica' (Simms et al. 1997). Sobre la base de los abundantes antecedentes sobre el registro cerámico de Chile y las provincias de Neuquén, Mendoza y La Pampa (Menghin, 1962; Durán, 2000; Berón, 2007; Hajduk et al. 2011; Sugrañes \& Franchetti, 2013), se plantea que estos tiestos habrían sido producidos en ámbitos lejanos al de recuperación, muy probablemente en Chile.

A nivel funcional, la baja frecuencia de material cerámico en $\mathrm{CH} 1$ es significativa, ya que es el sitio muestreado en forma más intensa en la región a nivel de superficie y estratigrafía. A modo de ejemplo, al comparar este contexto con otros ubicados en distintos puntos de la cuenca del arroyo Buta Có, a poca distancia de $\mathrm{CH} 1$, se observa un marcado contraste, ya que estos últimos presentan elevadas densidades de material cerámico en superficie (Chidiak, 2013). En forma preliminar, la variabilidad espacial del registro muestra una asociación del material cerámico con el curso del arroyo, a ca. $4 \mathrm{~km}$ de $\mathrm{CH} 1$, en contextos probablemente asociados en forma más recurrente a la realización de actividades residenciales. Esta es una línea de evidencia que se profundizará para comprender el rol de los sitios dentro de las estrategias de movilidad y subsistencia a través del tiempo.

\section{DISCUSIÓN Y CONCLUSIONES}

Las evidencias presentadas para el sitio $\mathrm{CH} 1$ permiten iniciar y profundizar diversos temas en distintas escalas de análisis, comenzando en el nivel del sitio y su historia ocupacional a través del tiempo y alcanzando la escala macro-regional, en la cual se tratan temas como la extinción de la megafauna, el poblamiento humano inicial, la existencia de un hiato ocupacional macro-regional propuesto para el Holoceno medio y el modo de ocupación del sitio en el Holoceno tardío.

\section{Escala de sitio: formas de ocupación y tendencias temporales}

En la Fig. 10 se presentan los datos sobre proporciones de las distintas clases de evidencia recuperadas en la cuadrícula A1 de $\mathrm{CH} 1$,

Tabla 8. Frecuencia de reserva de corteza por materia prima lítica en los artefactos de la cuadrícula A1.

\begin{tabular}{|c|c|c|c|c|c|c|}
\hline Materia prima & $0 \%$ & $25 \%$ & $50 \%$ & $75 \%$ & $100 \%$ & Total \\
\hline obsidiana & $236(66,11)$ & $36(10,08)$ & $30(8,40)$ & $9(2,52)$ & $46(12,89)$ & $357(81,88)$ \\
\hline calcedonia & $38(74,51)$ & $8(15,69)$ & $2(3,92)$ & $2(3,92)$ & $1(1,96)$ & $51(11,70)$ \\
\hline sílice & $9(81,82)$ & - & $1(9,09)$ & - & $1(9,09)$ & $11(2,52)$ \\
\hline basalto & $14(82,36)$ & $1(5,88)$ & $1(5,88)$ & - & $1(5,88)$ & $17(3,90)$ \\
\hline Total & $297(68,12)$ & $45(10,32)$ & $34(7,80)$ & $11(2,52)$ & $49(11,24)$ & $436(100)$ \\
\hline
\end{tabular}

Referencias: entre paréntesis se indican los valores porcentuales. 
indicándose los niveles artificiales de extracción y los tres componentes analíticos. Se integran indicadores que no han sido detallados aquí, tales como los restos de micro-fauna y los coprolitos de carnívoros pequeños (i.e. zorros). El componente 1 abarca los niveles artificiales de extracción 6 a 9 y está fechado entre ca. 17.000 y 13.600 años cal AP (Tabla 1). Posee densidades muy bajas o nulas de todos los materiales, con la excepción de los coprolitos de carnivoros que alcanzan su abundancia máxima en el Pleistoceno final (p.e. 562 gramos en el nivel 6) y presentan una correlación negativa con la frecuencia de evidencias arqueológicas líticas $y$ de macro-fauna (respectivamente, $r=-0.53, p$. $0.17 ; r=-0.56, p .0 .14)$. Sobre esta base, y dado que esto no se explica por preservación diferencial, se sugiere una menor intensidad de ocupación de la cueva por parte de carnívoros en los lapsos con ocupación humana. Dada la baja densidad de evidencias arqueológicas en asociación a sectores disturbados de la estratigrafía, se adopta el criterio conservador de interpretar a este conjunto de los niveles artificiales 6-9 como redepositado, aunque esto debe ser profundizado.

El componente 2 abarca las extracciones 4 y 5 y presenta las primeras ocupaciones humanas firmes en $\mathrm{CH} 1$, con cinco fechas ubicadas entre ca. 11.700 y 10.400 años cal AP. Estadísticamente se distingue un número mínimo de dos ocupaciones (Fig. 6), lo cual es consistente con indicadores contextuales. Dadas las bajas frecuencias de evidencias óseas, líticas y vegetales, estos eventos presentarían una corta duración y muy baja intensidad de descarte (Fig. 10). Se registró la presencia de al menos dos estructuras lenticulares de fogón, huesos de guanacos con huellas antrópicas y evidencias que indican el procesamiento $y / 0$ descarte de recursos vegetales como Prosopis sp. y Retanilla patagonica. A nivel artefactual, se recuperaron desechos líticos correspondientes a estadios primarios y finales (reactivación/reformatización de instrumentos) de reducción. La fuerte representación de instancias finales de reducción y la ausencia de instrumentos son consistentes con repetidas estadías breves, que pueden funcionar en el marco de una estrategia tecnológica de 'aprovisionamiento de individuos' (sensu Kuhn, 2004). La materia prima más utilizada desde las primeras ocupaciones en adelante es la obsidiana local Cerro Huenul, determinada mediante el análisis geoquímico de artefactos de CH1 (Barberena et al. 2011, Tabla 4). Desde un punto de vista de aprendizaje del paisaje (Meltzer, 2003), este uso sistemático en las ocupaciones más tempranas no resulta sorprendente, ya que esta materia prima es un elemento conspicuo en la localidad Huenul. Por el contrario, resulta significativo el uso casi nulo de rocas silíceas en las ocupaciones tempranas. Estas rocas son de excelente calidad y también están localmente disponibles, aunque en muy baja frecuencia. Esto puede sugerir que no habían sido integradas en las redes de información sobre la estructura del paisaje y sus recursos.

El componente 4 se caracteriza por un fuerte incremento en la frecuencia de todos los indicadores de presencia humana, en particular en el nivel 3, que está fechado en ca. 1.500 años cal AP. A nivel faunístico se observa una baja diversidad taxonómica, con dominio del guanaco. Las tasas de descarte de instrumentos son muy bajas, lo cual en conjunto con la elevada frecuencia de desechos de reactivación/reformatización sugiere su descarte en otros emplazamientos. A excepción de las puntas de proyectil, se observa el predominio de instrumentos informales. Se han registrado camadas vegetales y estructuras, incluyendo un hornillo en la cuadrícula D1, que denotan un acondicionamiento del espacio no registrado en forma previa. En forma de hipótesis, se plantea la cronología del componente 4 como marco temporal para la realización del arte rupestre y la utilización de la cerámica.

A nivel de densidades de materiales, hay un fuerte contraste entre los componentes 2 y 4 , indicando una ocupación humana de mucha mayor intensidad en el Holoceno tardío en comparación con el Holoceno temprano. Sin embargo, la composición de los conjuntos líticos y faunísticos no varía en forma fundamental. Como se mencionó, a nivel faunístico los dos componentes presentan un predominio de guanaco, mientras que el resto de los taxa está representado en muy bajas proporciones. Hay tendencias, que no son contundentes, que podrían contradecir lo esperado a partir de los modelos regionales de intensificación de la subsistencia en el Holoceno tardío (Neme \& Gil, 2008b): los conjuntos del Holoceno temprano 


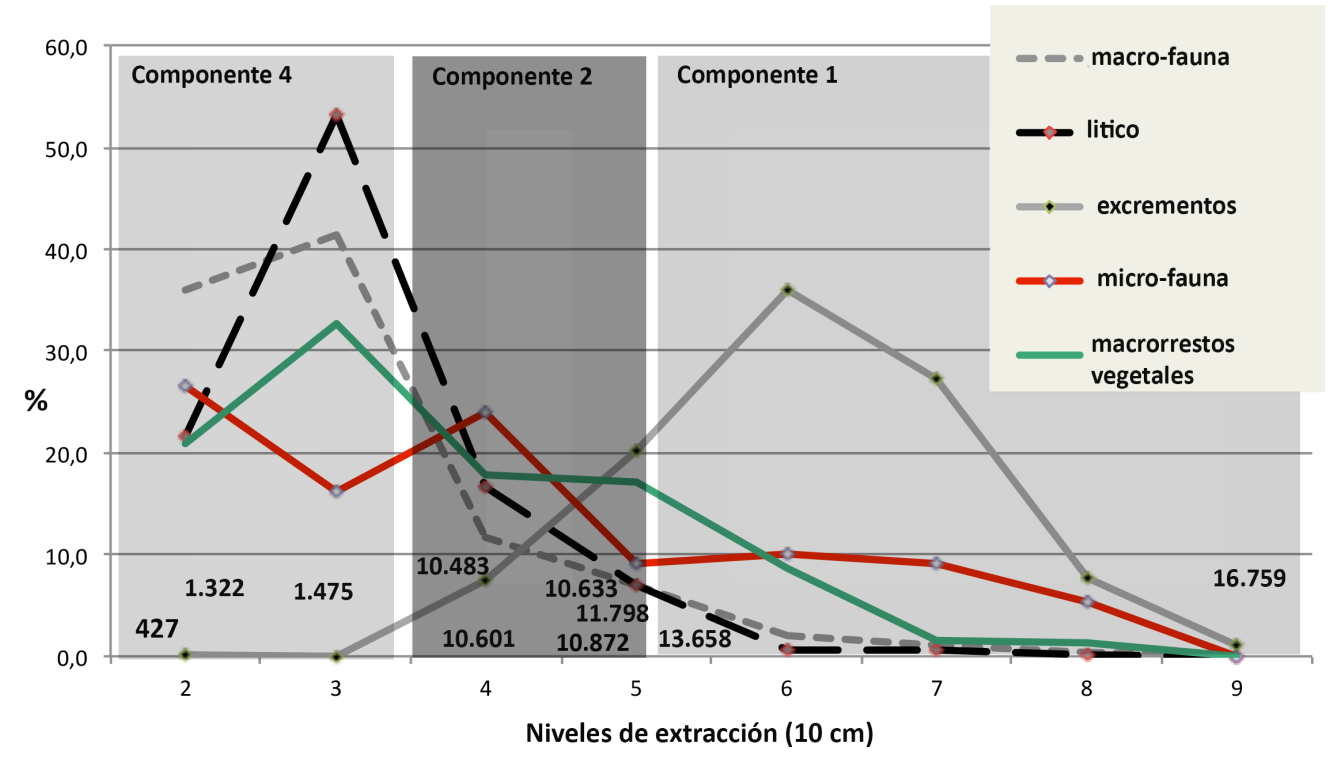

Fig. 10. Tasas de descarte de materiales en la cuadrícula A1.

evidencian una mayor intensidad de procesamiento de las carcasas de guanaco (cf. Lyman, 2005), así como también una mayor proporción de mamíferos pequeños, usualmente contemplados como de bajo ránking $e$ indicadores de dietas más amplias $e$ 'intensificadas'. El lítico evidencia repetidas actividades de reactivación de instrumentos que, a nivel general, no se habrían descartado in situ. Esto sugiere episodios recurrentes aunque breves de ocupación de $\mathrm{CH} 1$ a través del tiempo, con una mayor frecuencia en el Holoceno tardío.

Escala regional: Implicancias para el poblamiento humano de Patagonia septentrional

En $\mathrm{CH} 1$ se registró un hiato de ca. 3.000 años cal. entre la última presencia de megafauna y las primeras ocupaciones humanas, en consonancia con otros registros del sur de Mendoza y el extremo norte de Patagonia que muestran una ausencia de superposición temporal: Cueva Traful I (Crivelli Montero et al. 1993), Arroyo Malo 3 (Diéguez \& Neme, 2003), Manqui Malal (Praderio et al. 2012), Gruta El Manzano (Neme et al. 2011) y El Chacay (Neme \& Gil, 2008a) (Fig. 1). En este contexto, el registro aquí presentado respalda propuestas previas que indican que la megafauna había desaparecido en amplias regiones de Patagonia andina en forma previa a la colonización humana, sugiriendo la relevancia de las condiciones climáticas y ecológicas como causante principal de dichas extinciones (Borrero, 2009). Sin embargo, no todos los casos se ajustan a esta situación, ya que hay evidencias de fortaleza diversa que indican coexistencia en el norte de Patagonia -a ambos lados de los Andes- y en Cuyo: Monte Verde (Dillehay, 1997), Gruta del Indio (García, 2003), Alero El Trébol (Hajduk et al. 2011), entre otros (Fig. 1). Esto puede responder a la distribución geográfica de las poblaciones humanas tempranas que, desde un nivel teórico y empírico, se estima que fue muy heterogénea.

La fecha $\mathrm{CH} 1.10$ de $10.155 \pm 98$ (rango calibrado: 12.031-11.617 años AP) amplía la profundidad temporal de la presencia humana en el actual territorio de la provincia del Neuquén. El componente 2 de $\mathrm{CH} 1$, ubicado entre ca. 10.000 y 9.000 años ${ }^{14} \mathrm{C}$ AP, se alinea con los conjuntos tempranos de otros sitios en reparos que indican un pulso de presencia humana en el Holoceno temprano: Cueva Traful I (10.689 - 10.225 AP), Cueva Epullán Grande (11.772 - 11.178 AP), Cuyín Manzano (11.200 - 9.886 AP), Arroyo Corral II (11.825 - 11.210 AP) y Arroyo Malo 3 (10.169 - 9.920 AP) (Ceballos, 1982; Crivelli Montero et al. 1993, 1996; Neme \& Gil, 2008a; Arias Cabal et al. 2012). Este cuerpo de datos sugiere una señal de presencia humana inicial que 
es pene-contemporánea en espacios ampliamente separados entre sí y es compatible con una dispersión humana hacia espacios marginales desde nodos geográficos colonizados con anterioridad, que incluirían aquellos espacios con evidencias de interacción con la megafauna.

En la macro-región de Cuyo, adyacente al norte de Neuquén, el Holoceno medio se asocia a una disminución notoria en la abundancia de fechados radiocarbónicos que ha sido vinculada a las condiciones de aridez registradas para la época (Neme \& Gil, 2008a). En CH1 se registra un amplio hiato temporal (Hiato 2) que se extiende entre $9.000 \mathrm{y}$ 5.517 años radiocarbónicos y que abarca a los dos sectores excavados de la cueva (Fig. 2). Este hiato se asocia a una ausencia de sedimentos, ya que en la cuadrícula A1 hay continuidad estratigráfica inmediata entre los componentes 2 y 4 . Esto puede deberse a una ausencia de ocupaciones o a procesos erosivos, dando un significado ocupacional o tafonómico a este patrón. Contemplando a los humanos como el principal agente de sedimentación en $\mathrm{CH} 1$ durante el Holoceno, planteamos que la limitada presencias de sedimentos asignables al Holoceno medio es producto de una ocupación humana de muy baja intensidad y no de procesos de destrucción tafonómica. Por otra parte, al ubicar a $\mathrm{CH} 1$ en un marco regional en relación en torno a 4.800-3.900 AP para los sitios Hermanos Lazcano y Aquihuecó, ubicados hacia el sur de CH1 (Della Negra et al. 2009), se observa una delimitación del hiato temporal. Esta situación contrasta con el registro del centro de Neuquén, en particular Cueva Haichol, que presentaría continuidad de fechas en este período (Fernández, 1988-1990; Fernández \& Panarello, 2001).

El componente 4 es asignado a los últimos 1.600 años, instancia en la cual se plantea la ocupación humana de la mayor parte de los espacios disponibles en escala macro-regional, incluyendo aquellos más marginales desde un punto de vista ecológico, como el campo volcánico La Payunia en el sur de Mendoza, cuyo bloque temprano de ocupaciones se sitúa en torno a 2.000 años AP (Gil, 2006; Salgán, 2012). Recientemente hemos publicado un modelo biogeográfico enfocado en instancias de ocupación efectiva regional (sensu Borrero, 1989-1990), que estratifica el espacio del norte de Neuquén en función de sus aptitudes para el uso humano (Barberena, 2013). En el mismo, se postula que los espacios en torno a $\mathrm{CH} 1$ conformarían un ámbito marginal en las redes de circulación e instalación humana, en forma semejante a lo registrado para La Payunia (Salgán, 2012). Este modelo regional genera expectativas para las escalas espaciales pequeñas, de localidad y sitio. En forma preliminar, sugerimos que las evidencias aquí presentadas para $\mathrm{CH} 1$ son consistentes con un uso humano discontinuo, breve y poco intenso, y esto es coherente con un modelo de marginalidad regional. Por lo tanto, proveen un primer paso para contrastar el modelo regional.

\section{Perspectivas}

Se ha presentado una síntesis de la información disponible para el sitio arqueológico $\mathrm{CH} 1$, incluyendo resultados estratigráficos y cronológicos sobre los cuales se construye una secuencia histórica conformada por tres componentes analíticos que constituyen el primer bloque de información crono-estratigráfica disponible en escala regional. Estos resultados permiten comparar en forma preliminar las ocupaciones humanas registradas para el Holoceno temprano y el Holoceno tardío, así como ampliar el registro espacial del hiato propuesto para el Holoceno medio (Barberena, 2015).

El cuerpo de información presentado provee un modelo de la secuencia regional de ocupaciones, el cual será profundizado mediante una base de datos más amplia. Actualmente hay en desarrollo un conjunto de líneas de trabajo en el nivel del sitio y la región, mediante las cuales se espera profundizar y complejizar el panorama aquí propuesto. Se está generando una línea de reconstrucción paleoambiental y paleoclimática del noroeste de Patagonia, la cual permitirá contextualizar la historia de las ocupaciones humanas en términos de la estructura del paisaje y los recursos. La misma se basa sobre múltiples indicadores (isótopos estables, sedimentología, polen, macrorestos vegetales, tefrocronología) de registros sedimentarios de lagunas altoandinas, vegas, paleomadrigueras de roedores y sitios arqueológicos. Esto se articula con un estudio de la ecología isotópica regional de especies animales y vegetales, que proveerá la base para reconstrucciones paleodietarias. Asimismo, se encuentra en proceso de análisis el material de las 
demás cuadrículas excavadas en $\mathrm{CH} 1$, así como también las representaciones rupestres. En escalas más amplias, se están desarrollando estudios distribucionales, tecnológicos y composicionales de evidencias líticas y cerámicas. Estas y otras líneas de trabajo, en conjunto con una ampliación de las prospecciones hacia espacios de mayor altitud ubicados hacia el oeste, permitirán avanzar en nuestro conocimiento de la arqueología del extremo noroeste de Patagonia.

\section{AGRADECIMIENTOS}

Esta publicación es producto del proyecto Proyecto PICT Bicentenario 2010-1856 (Agencia Nacional de Promoción de la Ciencia y la Tecnología, Argentina). Se agradece al CONICET por su apoyo mediante un subsidio para Radicación de Investigadores en el Interior (Universidad Nacional de Cuyo, Mendoza) y a la Agencia Nacional de Promoción de la Ciencia y la Tecnología mediante su contribución mediante el proyecto PICT 2012-1976 (dirigido por Carina Llano). A Mónica Berón (Proyecto UBCAyT F-581) por su generosa colaboración. Especialmente a las autoridades de Buta Ranquil y Barrancas (provincia del Neuquén), que hacen posible nuestro trabajo: Juvenal Urrutia, Paulina Valenzuela, Hugo Zalazar, Marcela Carrera, Nora Vázquez, Gabriel Barros y Roque Díaz. Lo mismo cabe a numerosos amigos en el norte de Neuquén: Zidy Muñoz, Raúl "Yagui", Nelson y Héctor Vázquez. A Claudia Della Negra, Estela M. Cúneo, Liliana Martínez y Pablo Azar (Subsecretaría de Cultura de la Provincia del Neuquén) por la ayuda logística y los consejos brindados a través del tiempo. A Gia Lazzari y Catalina Rodillo (Universidad SEK, Chile) por su ayuda en el campo. A Valeria Cortegoso por todos sus consejos. A Ulyses Pardiñas y Pablo Teta por su aporte con el análisis de micro-fauna. Rafael Goñi brindó información sobre los trabajos originales en Huenul. M. Reguero permitió acceder a la colección paleontológica del Museo de Ciencias Naturales de La Plata. N. Toledo y G. Rougier ofrecieron importantes comentarios referidos al registro paleontológico. A Mitzi de Martino y el National Science Foundation AMS Laboratory, University of Arizona (EEUU). A los evaluadores anónimos y al comité editorial de Magallania por las importantes observaciones brindadas.

\section{BIBLIOGRAFÍA}

Andrefsky, W. (1994). Raw-material availability and the organization of technology. American Antiquity, 59, 21-34.

Arias Cabal, P., Hajduk, A., Crivelli, E., Chauvin, A., Albornoz, A., Armendáriz, A., Caracotche, S., Cueto, M., Fernández, M., Fernández, P., Lezcano, M., Palacio, E., Tapia, J., Tammone, M., Teira, L.C., \& Vallejo, J. (2012). El poblamiento temprano del noroeste de la Patagonia argentina. Trabajos desarrollados durante 2011. En Informes y Trabajos. Excavaciones en el exterior 2011 - 09 (pp. 19-41). Madrid: Ministerio de Educación, Cultura y Deporte de España.

Aschero, C. A. (1983). Ensayo para una clasificación morfológica de artefactos líticos aplicada a estudios tipológicos comparativos. Apéndices A-C. Revisión. Ergología y Tecnología, FFyL-UBA, Buenos Aires. Ms.

Aschero, C. A. (1988). Pinturas rupestres, actividades y recursos naturales: un encuadre arqueológico. En H. Yacobaccio (Ed.), Arqueología Contemporánea Argentina. Actualidad y Perspectivas (pp. 109-145). Buenos Aires: Ediciones Búsqueda.

Aschero, C. A. \& S. Hocsman. (2004). Revisando cuestiones tipológicas en torno a la clasificación de artefactos bifaciales. En A. Acosta, D. Loponte y M. Ramos (compiladores), Temas de arqueología. Análisis lítico (pp. 7-25). Luján: Universidad Nacional de Luján.

Barberena, R. (2013). Biogeografía, competencia y demarcación simbólica del espacio: modelo arqueológico para el norte de Neuquén. Intersecciones en Antropología, 14, 367-382.

Barberena, R. (2014). Discordancias y discontinuidades en Patagonia septentrional: crono-estratigrafía de Cueva Huenul 1 (Neuquén, Argentina). En V. Cortegoso, V. Durán \& A. Gasco (Eds.), Arqueología de ambientes de altura en Mendoza y San Juan (pp. 203-219). Mendoza: EDIUNC, Universidad Nacional de Cuyo.

Barberena, R. (2015). Cueva Huenul 1 Archaeological Site (Northwestern Patagonia, Argentina): Initial Colonization and mid-Holocene Demographic Retraction. Latin American Antiquity, en prensa.

Barberena, R., Hajduk, A., Gil, A., Neme, G., Durán, V., Glascock, M. D., Giesso, M., Borrazzo, K., Pompei, M. P., Salgán, M. L., Cortegoso, V., Villarosa, G., \& Rughini, A. (2011). Obsidian in the south-central Andes: geological, geochemical, and archaeological 
assessment of north Patagonian Sources (Argentina). Quaternary International, 245(1), 25-36.

Barberena, R., Pompei, M. P., Otaola, C., Gil, A., Neme, G., Borrazzo, K., Durán, V. \& Hoguin, R. (2010). Pleistocene-Holocene Transition in Northern Patagonia: Evidences from Cueva Huenul 1 (Neuquén, Argentina). Current Research in the Pleistocene, 27, 5-8.

Behrensmeyer, A. K. (1978). Taphonomic and ecologic information from bone weathering. Paleobiology, 4, 150162.

Beltrame, M. O., Fugassa, M. H., Barberena, R., Udrizar Sauthier, D. E., \& Sardella, N. H. (2013). New record of anoplocephalid eggs collected from rodent coprolites from two archaeological sites of Patagonia, Argentina. Parasitology International, 62, 431-434.

Beltrame, O., Sardella, N., Fugassa, M., \& Barberena, R. (2011). Paleoparasitological analysis of rodent coprolites from the archaeological site Cueva Huenul 1, Patagonia (Argentina). Memorias do Instituto Oswaldo Cruz, 107(5), 604-608.

Bennett, J. L. (1999). Thermal Alteration of Buried Bone. Journal of Archaeological Science, 26(1), 1-8.

Berón, M. A. (2007). Integración de evidencias para evaluar dinámica y circulación de poblaciones en las fronteras del río Colorado. En F. Morello, A. Prieto, M. Martinic \& G. Bahamonde (Eds.), Arqueología de Fuego-Patagonia. Levantando piedras, desenterrando huesos... y develando arcanos (pp. 173-188). Punta Arenas: CEQUA.

Borrero, L. A. (1989-1990). Evolución cultural divergente en la Patagonia austral. Anales del Instituto de la Patagonia (Serie Cs. Sociales) 19, 133-139.

Borrero, L. A. (2009). The Elusive Evidence: The Archaeological Record of the South American Extinct Megafauna. En: G. Haynes (Ed.), American Megafaunal Extinctions at the End of the Pleistocene (pp. 145-68). Dordrecht: Springer Science.

Bronk Ramsey, C. (2009). Bayesian analysis of radiocarbon dates. Radiocarbon, 51(1), 337-360.

Bronk Ramsey, C. (2013). 2013 OxCal v. 4.2.3.

Campbell, R. \& Quiroz, D. (2015). Chronological database for Southern Chile (35 $\left.30^{\prime}-42^{\circ} \mathrm{S}\right), \sim 33000 \mathrm{BP}$ to present: Human implications and archaeological biases. Quaternary International, 356, 39-53

Capparelli, A \& Lema, V. (2011). Recognition of post-harvest processing of algarrobo (Prosopis spp.) as food from two sites of Northwestern Argentina: an ethnobotanical and experimental approach for desiccated macroremains. Archaeological and Anthropological Sciences, 3, 71-
92.

Ceballos, R. (1982). El sitio Cuyín Manzano. Serie Estudios y Documentos, 9, 1-66.

Chidiak, M. (2013). Tecnología cerámica en el Noreste de Neuquén (Depto. Pehuenches): un enfoque preliminar. En J.R. Bárcena \& S.E. Martin (Eds.), Libro de Resúmenes del XVIII Congreso Nacional de Arqueología Argentina (p. 563). La Rioja: Universidad Nacional de La Rioja, INCIHUSA-CONICET.

Cordero, A. (2009). Arqueofauna de las primeras ocupaciones de cueva Epullán Grande. Cuadernos de Antropología, 5, 159-188.

Cordero, A. (2011). Arqueofauna de las ocupaciones tempranas de Cueva Traful I, Provincia del Neuquén, Argentina. Arqueología, 17, 161-194.

Cordero, R., Lanzelotti, S. \& Panarello, H. (2002). INGEIS Radiocarbon Laboratory Dates IV. Radiocarbon, 44(1), 181-193.

Crivelli Montero, E., Curzio, D. \& Silveira, M. (1993). La estratigrafía de la Cueva Traful I (Provincia del Neuquén). Praehistoria, 1, 17-160.

Crivelli Montero, E., Pardiñas, U., Fernández, M., Bogazzi, M., Chauvin, A., Fernández, V. \& Lezcano, M. (1996). La Cueva Epullán Grande (Provincia del Neuquén, Argentina). Praehistoria, 2, 17-160

Della Negra, C., Novellino, P., Pérez, I., Hajduk, A. \& Bernal, V. (2009). Investigaciones arqueológicas y bioarqueológicas en el sitio Aquihueco (Norpatagonia): nuevos resultados. En M. Salemme, F. Santiago, M. Alvarez, E. Piana, M. Vázquez \& E. Mansur (Eds.), Arqueología de la Patagonia. Una Mirada desde el ultimo confín. Tomo 2 (pp. 669-676). Ushuaia: Utopías.

Diéguez, S. \& Neme, G. (2003). Geochronology of the Arroyo Malo 3 Site and the First Human Occupations in North Patagonia in the Early Holocene. En L. Miotti, M. Salemme \& N. Flegenheimer (Eds.), Where the South Winds Blow. Ancient Evidence of Paleo South Americans (pp. 87-92). College Station: CSFA.

Dillehay, T. D. (1997). Monte Verde. A Late Pleistocene Settlement in Chile. Volume 2. Washington: Smithsonian Institution Press.

Dillehay, T. D. (ED.). (2010). Late Prehispanic and Hispanic Ceramics in the Purén, Lumaco, and Liucura Valleys, La Araucania, Chile. Nashville: Vanderbilt University Publications in Anthropology, Number 53.

Durán, V. 2000. Poblaciones Indígenas de Malargüe. Su Arqueología e Historia. Mendoza: CEIDER, Serie Libros 1 . 
Durán, V., Giesso, M., Glascock, M. D., Neme, G., Gil, A., \& Sanhueza, L. (2004). Estudio de fuentes de aprovisionamiento y redes de distribución de obsidiana durante el Holoceno Tardío en el sur de Mendoza (Argentina). Estudios Atacameños, 28, 25-43.

Erickson, D., Smith, B., Clarke, A., Sandweiss, D. \& Tuross, N. (2005). An Asian origin for a 10,000-year-old domesticated plant in the Americas. Proceedings of the National Academy of Sciences, 102, 18315-18320.

Escola, P. (2004). La expeditividad y el registro arqueológico. Chungará, 36(I), 49-60.

Fernández, F. J., Pardiñas, U. F. J., Teta, P., \& Barberena, R. (2011). Environmental stability during the PleistoceneHolocene transition in northwestern Patagonia? The small mammals of Cueva Huenul 1 as evidence. Current Research in the Pleistocene, 28, 154-156.

Fernández, F. J., Teta, P., Barberena, R. \& Pardiñas, U. F. J. (2012). Small mammal remains from Cueva Huenul 1, northern Patagonia, Argentina. Taphonomy and paleoenvironments since the Late Pleistocene. Quaternary International, 278, 22-31.

Fernández, J. (1974-1976). Estudios sobre el arte rupestre de la provincia del Neuquén. Anales de Arqueología y Etnología, XXIX-XXXI, 5-36.

Fernández, J. (1988-1990). La cueva de Haichol. Arqueología de los pinares cordilleranos del Neuquén. Anales de Arqueología y Etnología, 43-45.

Fernández, J. \& Panarello, H. (2001). Cazadores recolectores del Holoceno Medi y Superior de la cueva Haichol, región cordillerana del Neuquén, República Argentina. Relaciones de la Sociedad Argentina de Antropología, XXVI, 9-30.

Folguera, A., Bottesi, G. , Zapata, T. \& Ramos, V. A. (2008). Crustal collapse in the Andean backarc since $2 \mathrm{Ma}$ Tromen volcanic plateau, Southern Central Andes (36 $\left.40^{\circ}-37^{\circ} 30^{\prime} \mathrm{S}\right)$. Tectonophysics, 459, 140-160.

Franco, N. V. (2002). Estrategias de utilización de recursos líticos en la cuenca superior del río Santa Cruz (Argentina). Tesis Doctoral. Facultad de Filosofía y Letras, Universidad de Buenos Aires. Ms.

Garcia, A. (2003). On the coexistence of man and extinct Pleistocene megafauna at Gruta del Indio (Argentina). Radiocarbon, 45(1), 33-39.

Gil, A. F. (2006). Arqueología de La Payunia (Mendoza, Argentina). El poblamiento humano en los márgenes de la agricultura. Oxford: Archaeopress, BAR International Series 1477.

Gradin, C. J. (1978). Algunos aspectos del análisis de las manifestaciones rupestres. Revista del Museo
Provincial, 1, 120-133.

Gradin, C. J. (1997-1998). El arte rupestre del sur mendocino entre los siglos VIII y XV de la era ¿Un área de conflicto o de convivencia? Relaciones de la Sociedad Argentina de Antropología, XXII-XXIII, 7-23.

Grayson, D. K. (1984). Quantitative Zooarchaeology. Topics in the Analysis of Archaeological Faunas. Orlando: Academic Press.

Groeber, P. (1926). Toponimia araucana. GAEA. Anales de la Sociedad Argentina de Estudios Geográficos, II(1).

Hajduk, A., Albornoz, A. M. \& Lezcano, M. J. (2011). Espacio, cultura y tiempo: el corredor bioceánico desde la perspectiva arqueológica. En P. Navarro Floria \& W. Delrío (Eds.), Cultura y espacio. AraucaníaNorpatagonia (pp. 262-292). Bariloche: Universidad Nacional de Río Negro.

Hajduk, A. \& Cúneo, E. (2009). Representaciones rupestres en la cuenca del río Curi-Leuvú (departamento Chos Malal, provincia del Neuquén). Informe preliminar. En M. Salemme, F. Santiago, M. Álvarez, E. Piana, M. Vázquez \& M.E. Mansur (Eds.), Arqueología de Patagonia: una mirada desde el último confín. Tomo 1 (pp. 515526). Ushuaia: Utopías.

Kuhn, S. (2004). Upper Paleolithic raw material economies at Ücagizli cave, Turkey. Journal of Anthropological Archaeology, 23, 431-448.

Ladio, A. \& Lozada, M. (2009). Human ecology, ethnobotany and traditional practices in a rural population of the Monte region, Argentina: resilience and ecological knowledge. Journal of Arid Environments, 73, 222227.

Llano, C. (2011). Aprovechamiento de los Recursos Vegetales entre las Sociedades Cazadoras-Recolectoras del Sur de Mendoza. Bariloche: Tesis Doctoral, Universidad Nacional del COMAHUE.

Llano, C. \& Barberena, R. (2013). Explotación humana de especies vegetales en Patagonia septentrional: el registro arqueobotánico de Cueva Huenul 1 (Provincia de Neuquén, Argentina). Darwiniana N.S., 1(1), 5-19.

López, L., Pérez, A. \& Stern, C. R. (2009). Fuentes de aprovisionamiento y distribución de obsidianas en la Provincia del Neuquén, noroeste de la Patagonia Argentina. Intersecciones en Antropología, 10, 75-88.

Lyman, R. L. (2005). Analyzing cut marks: lessons from artiodactyl remains in the northwestern United States. Journal of Archaeological Science, 32, 1722-1732.

Martínez, J. G., Powell, J. E. \& Rodríguez, M. F. (2010). Dung Analysis and its Correlation with Three Different Species 
of Extinct Megafauna in the Southern Argentinian Puna. Current Research in the Pleistocene, 27, 197129.

Massone, M., Sánchez, M., Quiroz, D. \& Contreras, L. (Eds.). (2011). Cazadores recolectores costeros en la Región del Bío-Bío. Concepción: Museo de Historia Natural de Concepción, Ediciones Escaparate.

Meltzer, D. J. (2003). Lessons in landscape learning. En M. Rockman \& J. Steele (Eds.), Colonization of Unfamiliar Landscapes. The archaeology of adaptation (pp. 222-241). Londres: Routledge.

Menghin, O. (1962). Estudios de prehistoria Araucana. Acta Prehistórica, 3-4.

Mengoni Goñalons, G. L. (1999). Cazadores de guanacos de la estepa patagónica. Buenos Aires: Sociedad Argentina de Antropología.

Miller, K. W. P., Walker, P. L. \& O'Halloran, R. L. (1998). Age and sex-related variation in hyoid bone morphology. Journal of Forensic Sciences, 43(6), 1138-1143.

Movia, C., Ower, G. H. \& Pérez, C. E. (1982). Estudio de la vegetación natural de la Provincia del Neuquén. Neuquén: Ministerio de Economía y Hacienda, Secretaría de Recursos Naturales.

Narciso, V., Santamaría, G. \& Zanettini, J. C. M. (2004). Hoja Geológica 3769-I, Barrancas. Provincias de Mendoza y Neuquén. Buenos Aires: Instituto de Geología y Recursos Minerales, SEGEMAR. Boletín 253.

Neme, G. \& Gil, A. (2008a). Biogeografía humana en los Andes meridionales: tendencias arqueológicas en el sur de Mendoza. Chungará, 40(1) 5-18.

Neme, G. \& Gil, A. (2008b). Faunal Exploitation and Agricultural Transitions in the South American Agricultural Limit. International Journal of Osteoarchaeology, 18(3), 293-306.

Neme, G., Gil, A., Garvey, R., Llano, C., Zangrando, A., Franchetti, F., De Francesco, C. \& Michieli, C. (2011). El registro arqueológico de la Gruta de El Manzano y sus implicancias para la arqueología de Nordpatagonia. Magallania, 39(2), 243-265.

Páez, M., Quintana, F. \& Pérez, C. (2004). Biogeografía de las Regiones Áridas y Semiáridas entre los $35^{\circ}-39^{\circ} \mathrm{S}$, Argentina. Boletín de la Sociedad Argentina de Botánica, 39(3-4), 171-180.

Pérez, L. M., Toledo, N., De Iuliis, G., Bargo, S., \& Vizcaíno, S. F. (2010). Morphology and function of the hyoid apparatus of fossil xenarthrans (Mammalia). Journal of Morphology, 271, 1119-1133.
Pompei, M. P., Barberena, R., de Porras, M. E., Borrazzo, K., Rughini, A. \& Gil, A. (2012). Late Quaternary Ecosystems and Humans in Northern Patagonia (Neuquén, Argentina). En L. Miotti, M. Salemme, N. Flegenheimer \& T. Goebel (Eds.), Current Research in the Pleistocene. Southbound. Late Pleistocene Peopling of Latin America (pp. 187-190). College Station: CSFA, Texas A\&M University.

Praderio, A., Gil, A. \& Forasiepi, A. (2012). El registro de Megatherium (Xenarthra, Tardigrada) en Mendoza (Argentina): aspectos taxonómicos, cronológicos y paleoecologicos. Mastozoología Neotropical, 19, 279-291.

Re, A. (2010). Representaciones Rupestres en Mesetas Altas de la Provincia de Santa Cruz. Circulación de Información en Espacios de Uso Estacional. Tesis de Doctorado. Universidad de Buenos Aires, FFyL. Ms.

Reimer, P., Bard, E., Bayliss, A., Beck, J., Blackwell, P., Ramsey, C., Buck,... van der Plicht, J. (2013). INTCAL13 and MARINE13 Radiocarbon Age Calibration Curves 0-50,000 Years Cal BP. Radiocarbon, 55, 1859-1887.

Romero, G. \& Re, A. (2014). Representaciones rupestres del noreste de Neuquén (Depto. Pehuenches). Comechingonia, 18, 73-92.

Salgán, M. L. (2012). Organización tecnológica y biogeografía humana en La Payunia, sur de la provincia de Mendoza. Tesis Doctoral. La Plata: FCNyM, Universidad Nacional de La Plata. Ms.

Schobinger, J. (1985). Áreas intermedias o marginales. En J. Schobinger y C.J. Gradin (Eds.), Cazadores de la Patagonia y agricultores andinos. Arte rupestre de la Argentina (pp. 80-91). Madrid: Encuentro.

Simms, S. R., Bright, J. R. \& Ugan, A. (1997). Plain-Ware Ceramics and Residential Mobility: A Case Study From the Great Basin. Journal of Archaeological Science, 24, 779-792.

Stein, J. K. (1990). Archaeological stratigraphy. En N.P. Lasca \& J. Donahue (Eds.), Archaeological geology of North America (pp. 513-523). Washington: Geological Society of America, Centennial Special Volume 4.

Sugrañes, N. \& Franchetti, F. (2013). Antecedentes, problemas y perspectivas del análisis cerámico en el sur de Mendoza. En G. Neme \& A. Gil (Eds.), Paleoecología humana en el sur de Mendoza: Perspectivas arqueológicas (pp. 229-253). Buenos Aires: Sociedad Argentina de Antropología. 
